\title{
COMBINATORIAL HOMOTOPY. II
}

\author{
J. H. C. WHITEHEAD
}

1. Introduction. This paper is concerned with problems of realizability, which were discussed in (I) (i.e. [1]). ${ }^{1}$ In studying the realizability of chain mappings we use the system of relative homotopy groups, $\rho_{n}=\pi_{n}\left(K^{n}, K^{n-1}\right)$, of a (connected) complex, $K$, where $n \geqq 1$ and $\rho_{1}=\pi_{1}=\left(K^{1}\right)$. The chain groups are defined as the relative homology groups, $C_{n}=H_{n}\left(\widetilde{K}^{n}, \widetilde{K}^{n-1}\right)$, where $\tilde{K}$ is a universal covering complex of $K$. The groups $\rho_{n}$ appear to be more useful than $C_{n}$ in problems concerning geometrical realizability. On the other hand the chain groups are convenient in studying concrete problems. Moreover they provide a means of applying Theorem 3 in (I). A large part of the paper deals with the relations between the two systems of groups.

The system of relative homotopy groups, $\left\{\rho_{n}\right\}$, is a "group system," as defined in [8]. It is a special kind of group system because each group is "free" in one of three different senses. More precisely, $\rho_{1}$ is a free group, $\rho_{n}$ is a free $\pi_{1}(K)$-module if $n>2$ and $\rho_{2}$ is what we call a free crossed module. These conditions of freedom are important in realizability problems. We tentatively describe $\left\{\rho_{n}\right\}$ as a homotopy system. It is essentially the same as what was called a "natural system" on p. 1216 of [3], redefined in terms of relative homotopy groups and free crossed modules.

2. Crossed modules. It will be convenient to have a name for groups with the algebraic properties of relative homotopy groups, and to have proved some lemmas concerning them. We shall call such a group a crossed module, or, more explicitly, a crossed $\boldsymbol{\gamma}$-module or a crossed $(\gamma, d)$-module. ${ }^{2}$ By this we mean an additive, but not necessarily commutative, group, $\rho$, which is related as follows to a multiplicative group $\gamma$ :

(2.1) (a) $\rho$ admits $\gamma$ as a group of operators. ${ }^{3}$

An address delivered before the Princeton Meeting of the Society on November 2, 1946, by invitation of the Committee to Select Hour Speakers for Eastern Sectional Meetings; received by the editors September 22, 1948.

${ }^{1}$ Numbers in brackets refer to the references cited at the end of the paper.

${ }^{2}$ Anne Cobbe has pointed out to me that a crossed $(\gamma, d)$-module determines a $Q$-kernel, with $Q=\gamma / d \rho$ (see [10]), and that any $Q$-kernel has a representation as a crossed module. I learn from Saunders MacLane that crossed modules, under a different name, are defined in a forthcoming sequel to [10].

${ }^{3}$ I.e. to each $x \in \gamma$ corresponds an automorphism, $x: \rho \rightarrow \rho$, such that $x^{\prime}(x a)$ $=\left(x^{\prime} x\right) a$ and $x a=a$ if $x=1\left(x, x^{\prime} \subset \gamma, a \in \rho\right)$. We allow trivial operators (i.e. elements $x \in \gamma$ such that $x a=a$ for every $\left.a \in_{\rho}\right)$ other than $1 \in \gamma$. 
(b) There is a homomorphism, $d: \rho \rightarrow \gamma$, such that $d(x a)=x(d a) x^{-1}$ $(a \in \rho, x \in \gamma)$.

(c) $a+b-a=(d a) b$ for every pair, $a, b \subset \rho$.

Notice that any group, $\rho$, is a crossed $(\gamma, d)$-module, with a suitable choice of $\gamma$ and $d$. For example we may take $\gamma$ to be the group of inner automorphisms of $\rho$, and $d$ to be defined by (2.1c). Notice also that any Abelian group, $\rho$, which admits $\gamma$ as a group of operators is a crossed $(\gamma, d)$-module with $d \rho=1$.

Let $C$ be the group $\rho$ made Abelian and let $h$ be the natural homomorphism $h: \rho \rightarrow C$. Then $h^{-1}(0)$ is generated by the commutators, $a+b-a-b$, or, using (2.1c), $(d a) b-b$. Therefore $C$ may also be regarded as $\rho$ with the operators in $d \rho$ "neutralized." It follows from (2.1b) that $d \rho$ is an invariant sub-group of $\gamma$. Let $\bar{\gamma}=\gamma / d \rho$ and let $\bar{x} \in \bar{\gamma}$ be the co-set containing a given element $x \in \gamma$. Given $h a \in C$, we define $\bar{x}(h a)=h(x a)$. The transformation $\bar{x}: C \rightarrow C$, thus defined, is single-valued since the commutator sub-group of $\rho$ is transformed into itself by $x$ (and indeed by any automorphism). It is obvious that $\bar{x}: C \rightarrow C$ is an endomorphism; also that $1 h a=h a$ and $\bar{x}\left(\bar{x}^{\prime} h a\right)=\left(\bar{x} \bar{x}^{\prime}\right) h a$. Therefore $C$ is a $\bar{\gamma}$-module, i.e. an Abelian group which admits $\bar{\gamma}$ as a group of operators. Moreover $h$ is an operator homomorphism, in the sense that $h(x a)=\bar{x} h a$.

It follows from (2.1c) that $(d a) b=b$ if $b$ is in the center of $\rho$. That is to say the elements in $d \rho$ operate trivially on the centre of $\rho$, which may therefore be regarded as a $\bar{\gamma}$-module. It also follows from (2.1c), with $d a=1$, that $d^{-1}(1)$ is in the centre of $\rho$. Moreover it follows from (2.1b) that $d(x a)=1$ if $d a=1$, whence $d^{-1}(1)$ is transformed into itself by the operators in $\gamma$. Therefore $d^{-1}(1)$ may also be regarded as a $\bar{\gamma}$-module.

Since $d^{-1}(1)$ is in the centre of $\rho$ the group $\rho$ is a central extension of $d^{-1}(1)$ by $d \rho$. If it is also a trivial extension, i.e. if $d$ has a right inverse, $\theta: d \rho \rightarrow \rho(d \theta=1)$, it follows that $\rho$ is the direct sum $\rho=d^{-1}(1)$ $+\theta d \rho$. Since $h$ makes $\rho$ Abelian and since $d^{-1}(1)$ is already Abelian it follows that $h \mid d^{-1}(1)$ is then an isomorphism (into). Therefore we have:

LEMma 1. If $\rho$ is a trivial extension of $d^{-1}(1)$ by $d \rho$, then $d a=1$ and $h a=0$ together imply $a=0$.

In particular $h \mid d^{-1}(1)$ is an isomorphism (into) if $d \rho$ is a free group, since any extension by a free group is trivial. Therefore $h \mid d^{-1}(1)$ is an isomorphism (into) if $\gamma$ is a free group.

Let $\rho^{\prime}$ be a crossed $\left(\gamma^{\prime}, d^{\prime}\right)$-module and let $g: \gamma \rightarrow \gamma^{\prime}$ be a given homomorphism. By an operator homomorphism, $f: \rho \rightarrow \rho^{\prime}$, associated with $g$ 
we mean a homomorphism such that

(a) $d^{\prime} f=g d$,

(b) $f(x b)=(g x) f b \quad(x \in \gamma, b \in \rho)$.

We shall describe $f$ as an operator isomorphism ${ }^{4}$ if, and only if, it and $g$ are both isomorphisms. Notice that this definition applies to ordinary modules, with $d \rho=1, d^{\prime} \rho^{\prime}=1$, in which case $(2.2 \mathrm{~b})$ is the operative condition. Notice also that, if there is no $x^{\prime} \in \gamma^{\prime}$ such that $x^{\prime} f b=f b$ for every $b \in \rho$, then it follows from (2.2b) that the same operator homomorphism, $\rho \rightarrow \rho^{\prime}$, cannot be associated with each of two different homomorphisms $\gamma \rightarrow \gamma^{\prime}$. This is the case, for example, if $\rho^{\prime}$ is a free $\gamma^{\prime}$-module and $f \rho$ contains a basis element of $\rho^{\prime}$.

We now define what we call a free crossed $\gamma$-module. ${ }^{5}$ Let $\left\{\alpha_{i}\right\}$ be any indexed ${ }^{6}$ aggregate, let $\gamma$ be a given group and let an arbitrary element $x_{i} \in \gamma$ be associated with each $\alpha_{i}$. We define an additive group, $\rho$, by means of "symbolic" generators and relations. The generators shall be all triples $\left(+, x, \alpha_{i}\right)$, where $x \in \gamma$, together with their "negatives" $\left(-, x, \alpha_{i}\right)$, We write $\left(+, x, \alpha_{i}\right)$ as $\left(x, \alpha_{i}\right)$ and $\left(-, x, \alpha_{i}\right)$ as $-\left(x, \alpha_{i}\right)$. The relations shall be

$$
\left(x, \alpha_{i}\right)+\left(y, \alpha_{j}\right)-\left(x, \alpha_{i}\right)=\left(x x_{i} x^{-1} y, \alpha_{j}\right),
$$

for all pairs $x, y \subset \gamma$ and all values of $i, j$, together with the "trivial" relations, $\left(x, \alpha_{i}\right)-\left(x, \alpha_{i}\right)=0$ and $-\left(x, \alpha_{i}\right)+\left(x, \alpha_{i}\right)=0$.

Since

$$
\begin{aligned}
\left(z x, \alpha_{i}\right)+\left(z y, \alpha_{j}\right)-\left(z x, \alpha_{i}\right) & =\left(z x x_{i} x^{-1} z^{-1} z y, \alpha_{j}\right) \\
& =\left(z x x_{i} x^{-1} y, \alpha_{j}\right)
\end{aligned}
$$

the transformation $\left(x, \alpha_{i}\right) \rightarrow\left(z x, \alpha_{i}\right)$, of generators, determines an endomorphism, $z: \rho \rightarrow \rho(z \in \gamma)$. Obviously $z a=a$ if $z=1, z^{\prime}(z a)=\left(z^{\prime} z\right) a$, where $a \in \rho$. Therefore $\rho$ admits $\gamma$ as a group of operators. Similarly a homomorphism $d: \rho \rightarrow \gamma$, which satisfies (see [2, p. 421]) (2.1b), is defined by $\left(x, \alpha_{i}\right) \rightarrow x x_{i} x^{-1}$. Then (2.1c) follows (see [2, p. 421]) from (2.3) and the trivial relations. Therefore $\rho$ is a crossed $(\gamma, d)$-module.

Let $a_{i} \in \rho$ be the element which corresponds to the generator (1, $\left.\alpha_{i}\right)$. We shall call the set of elements $\left\{a_{i}\right\}$ a basis for $\rho$. Any element in

${ }^{4} \mathrm{An}$ isomorphism, without qualification, will always mean an isomorphism onto.

5 This is a group of the sort defined on p. 421 of [2]. A correspondence with Saunders MacLane has helped to clarify the presentation.

${ }^{6}$ The set of values taken by $i$ may have any cardinal number. The elements $\alpha_{i}$ are not elements of the group, $\rho$, which is to be defined. An element of $\rho$ is an equivalence class of "words," written as sums, of the "symbolic" generators. $\alpha_{i} \neq \alpha_{j}$ if $i \neq j$. 
$\rho$ is of the form

$$
\epsilon_{1} x_{1} a_{i_{1}}+\cdots+\epsilon_{n} x_{n} a_{i_{n}}
$$

where $\epsilon_{i}= \pm 1, x_{i} \in \gamma$, and repetitions are allowed.

We shall describe a crossed module, $\rho^{\prime}$, as free if, and only if, it is operator isomorphic to a crossed module, $\rho$, of the sort just defined. This being so, a set of elements will be called a basis for $\rho^{\prime}$ if, and only if, it is the image of $\left\{a_{i}\right\}$ in an operator isomorphism $\rho \rightarrow \rho^{\prime}$. In general there will be many operator isomorphisms, $\rho \rightarrow \rho^{\prime}$, and hence many bases for $\rho^{\prime}$.

We now prove a lemma, which is fundamental in realizability problems. Let $\rho$ be a free crossed $(\gamma, d)$-module and $\rho^{\prime}$ an arbitrary crossed $\left(\gamma^{\prime}, d^{\prime}\right)$-module. Let $g: \gamma \rightarrow \gamma^{\prime}$ be an arbitrary homomorphism and let $\left\{a_{i}\right\}$ be a basis for $\rho$. With each basis element $a_{i}$ we associate an element $b_{i}^{\prime} \in \rho^{\prime}$, which satisfies the condition $d^{\prime} b_{i}^{\prime}=g d a_{i}$, but is otherwise arbitrary.

LEMмA 2. There is a unique operator homomorphism, $f: \rho \rightarrow \rho^{\prime}$, associated with $g$, such that $f a_{i}=b_{i}^{\prime}$ for each value of $i$.

We assume, as we obviously may, that $\rho$ is defined, as above, in terms of symbolic generators $\left(x, \alpha_{i}\right)$ and that $a_{i}$ is the element corresponding to $\left(1, \alpha_{i}\right)$. Then $d^{\prime} b_{i}^{\prime}=g d a_{i}=g x_{i}$ and it follows from (2.1c), in $\rho^{\prime}$, that

$$
\begin{aligned}
(g x) b_{i}^{\prime}+(g y) b_{j}^{\prime}-(g x) b_{i}^{\prime} & =(g x) d^{\prime} b_{i}^{\prime}(g x)^{-1}(g y) b_{j}^{\prime} \\
& =g\left(x x_{i} x^{-1} y\right) b_{j}^{\prime} .
\end{aligned}
$$

Therefore the transformation $\left(x, \alpha_{i}\right) \rightarrow(g x) b_{i}^{\prime}$, of generators, determines a homomorphism, $f: \rho \rightarrow \rho^{\prime}$, which obviously satisfies (2.2b). Also $f a_{i}=b_{i}^{\prime}$ and $d^{\prime} f a_{i}=g d a_{i}$. Therefore it follows from (2.1b) that

$$
\begin{aligned}
d^{\prime} f x a_{i} & =d^{\prime}\left\{(g x) f a_{i}\right\}=(g x)\left(d^{\prime} f a_{i}\right)(g x)^{-1} \\
& =g\left\{x\left(d a_{i}\right) x^{-1}\right\}=g d x a_{i} .
\end{aligned}
$$

Since $\rho$ is generated by $\left\{x a_{i}\right\}$ it follows that (2.2a) is satisfied. Similarly $f$ is uniquely determined by the condition $f a_{i}=b_{i}^{\prime}$, and the lemma is proved.

We conclude the section with two "extreme" examples of a free crossed module, $\rho$. First let $d \rho=1$. Then (2.3) merely expresses the fact that $\rho$ is commutative, and $\rho$ will be called a free $\gamma$-module. In this case we can collect all the terms $\epsilon_{\lambda} x_{\lambda} a_{i_{\lambda}}$ in (2.4), which have the same $i_{\lambda}$, and write (2.4) as

$$
r_{1} a_{j_{1}}+\cdots+r_{k} a_{j_{k}}
$$


where $j_{1}, \cdots, j_{k}$ are distinct and $r_{1}, \cdots, r_{k}$ are in the group ring, $R$, of $\gamma$. This expression is unique since, obviously, $\left\{x a_{i}\right\}$ are free generators of $\rho$. Therefore $\rho$ is a free $R$-module, having $\left\{a_{i}\right\}$ as a basis. In any case $h \rho$ is obviously a free $\bar{\gamma}$-module, having $\left\{h a_{i}\right\}$ as a basis, where $h: \rho \rightarrow h \rho$ makes $\rho$ Abelian. Notice that, in consequence of this fact, there is no relation of the form $m_{1} a_{i_{1}}+m_{2} a_{i_{2}}+\cdots=0$, in $\rho$, where $m_{1}, m_{2}, \cdots$ are nonzero integers and $i_{1}, i_{2}, \cdots$ are distinct.

A free Abelian group,${ }^{7}$ which admits $\gamma$ as a group of operators, is not necessarily a free $\gamma$-module. Therefore a crossed $(\gamma, d)$-module is not necessarily free just because (2.1c) is a complete set of relations between a set of generating elements $a, b, \cdots$.

Secondly let $\gamma$ be a free group and, in the original definition of a free crossed module, let $\left\{x_{i}\right\}$ be a set of free generating elements of $\gamma$ (inverses not included). Let $x=x_{i_{1}}^{\epsilon_{1}} \cdots x_{i_{n}}^{\epsilon_{n}}\left(e_{\lambda}= \pm 1\right)$. Then it follows from (2.3), and their "negatives," which are consequences of (2.3) and the trivial relations, that

$$
\begin{aligned}
\left(x x_{i}, \alpha_{j}\right) & =\left(x, \alpha_{i}\right)+\left(x, \alpha_{j}\right)-\left(x, \alpha_{i}\right), \\
\left(x x_{i}^{-1}, \alpha_{j}\right) & =-\left(x, \alpha_{i}\right)+\left(x, \alpha_{j}\right)+\left(x, \alpha_{i}\right) .
\end{aligned}
$$

Therefore it follows by induction on $n$ that $\rho$ is generated by $\left\{a_{i}\right\}$, without the help of the operators in $\gamma$. Therefore the right inverse, $d^{-1}: \gamma \rightarrow \rho$, of $d$, which is given by $d^{-1} x_{i}=a_{i}$, is onto $\rho$. Therefore $d: \rho \approx \gamma$. Thus $\rho$ is an ordinary free group, which is freely generated by $\left\{a_{i}\right\}$. Also $\gamma$ written additively and operating on itself according to the rule $x y=x+y-x(x, y \subset \gamma)$ is a free $(\gamma, 1)$-module. A set of free generating elements will be called a basis for $\gamma$, whether the multiplicative or the additive notation is used.

3. A lemma on crossed homomorphisms. Let $G$ be an additive group, which need not be Abelian, and let $\gamma$ be a multiplicative group, which operates on $G$. A crossed homomorphism, $h: \gamma \rightarrow G$, is a map such that

$$
h(x y)=h x+x h y \quad(x, y \subset \gamma) .
$$

We shall generally be presented with a group $G$, a group, $\gamma^{\prime}$, which operates on $G$, and a homomorphism $f: \gamma \rightarrow \gamma^{\prime}$. In this case a crossed homomorphism, $h: \gamma \rightarrow G$, will mean the same as before, with $x a=(f x) a(x \in \gamma, a \in G)$, and $h$ will be called a crossed homomorphism associated with $f$. Notice that, writing $x=1$ in (3.1), we have $h(1)=0$ and writing $x=y^{-1}$ we have $h y^{-1}=-y^{-1} h y$.

\footnotetext{
${ }^{7}$ E.g. one in which $\gamma$ operates trivially, or a left ideal in the group ring of $\gamma$.
} 
As an example, which occurs in $\S 10$ below, let $f, f^{\prime}: \gamma \rightarrow G$ be two homomorphisms and let us write $a b=a+b-a(a, b \subset G)$. Let $\theta: G \rightarrow G$ be a fixed endomorphism and let $h: \gamma \rightarrow G$ be given by

$$
h x=\theta f^{\prime} x-f x .
$$

Then

$$
\begin{aligned}
h(x y) & =\theta f^{\prime}(x y)-f(x y) \\
& =\theta f^{\prime} x+\theta f^{\prime} y-f y-f x \\
& =\left(\theta f^{\prime} x-f x\right)+f x+\left(\theta f^{\prime} y-f y\right)-f x \\
& =h x+(f x) h y .
\end{aligned}
$$

Therefore $h$ is a crossed homomorphism associated with $f$.

Let $\gamma$ be a free group, which operates on $G$, and let $\left\{x_{i}\right\}$ be a basis for $\gamma$. With each $x_{i}$ we associate an arbitrary element $a_{i} \in G$.

Lemma 3. There is a unique crossed homomorphism, $h: \gamma \rightarrow G$, such that $h x_{i}=a_{i}$ for each value of $i$.

Any element $y \in \gamma$ is uniquely represented as a product

$$
y=x_{i_{1}}^{\epsilon_{1}} \cdots x_{i_{n}}^{\epsilon_{n}} \quad\left(\epsilon_{i}= \pm 1\right),
$$

which is reduced, in the sense that $\epsilon_{j+1}=\epsilon_{j}$ if $i_{j+1}=i_{j}$ (i.e. no cancellation is possible). We define $h: \gamma \rightarrow G$ by

$$
h y=\epsilon_{1} y_{1} a_{i_{1}}+\cdots+\epsilon_{n} y_{n} a_{i_{n}},
$$

where $y_{1}=1$ if $\epsilon_{1}=1$ and

$$
\begin{aligned}
y_{j} & =x_{i}^{\epsilon_{1}} \cdots x_{i_{j-1}}^{\epsilon_{j-1}} & \text { if } & \epsilon_{j}=+1 \\
& =x_{i_{1}} \cdots x_{i_{j}}^{\epsilon_{j}} & \text { if } & \epsilon_{j}=-1 .
\end{aligned}
$$

Then $h x_{i}=a_{i}, h x_{i}^{-1}=-x_{i}^{-1} a_{i}$. It follows from (3.2) and (3.3) that

$$
\begin{aligned}
& h\left(z x_{i}^{i}\right)=h z+z a_{i} \quad \text { if } \quad \epsilon=+1 \\
& =h z-z x_{i}^{-1} a_{i} \quad \text { if } \quad \epsilon=-1 \\
& =h z+z h x_{i}^{\epsilon} \quad \text { if } \epsilon= \pm 1,
\end{aligned}
$$

for any $z \in \gamma$. Hence, assuming that $h(x y)=h x+x h y$, for a given $x \in \gamma$, we have

$$
\begin{aligned}
h\left(x y x_{i}^{\epsilon}\right) & =h(x y)+x y h x_{i}^{\epsilon} \\
& =h x+x\left(h y+y h x_{i}^{\epsilon}\right) \\
& =h x+x h\left(y x_{i}^{\epsilon}\right) .
\end{aligned}
$$


It follows from induction on $n$ that (3.1) is satisfied. Therefore $h$ is a crossed homomorphism.

Let $h: \gamma \rightarrow G$ be any crossed homomorphism such that $h x_{i}=a_{i}$. Then it follows from (3.1) and induction on $n$ that $h$ is given by (3.2) and (3.3). Therefore $h$ is unique and the lemma is proved.

4. Homotopy systems. Let $\rho=\left\{\rho_{n}\right\}(n=1,2, \cdots)$ be a family of groups, together with a "boundary operator," $d$, which is a family of homomorphisms, $d_{r}: \rho_{r} \rightarrow \rho_{r-1}(r \geqq 2)$, such that $d d=0$ (i.e. $d_{r} d_{r+1} \rho_{r+1}$ is the neutral element in $\rho_{r-1}$ ). The group $\rho_{n}$ shall be multiplicative if $n=1$ and additive if $n>1$, though possibly noncommutative if $n=2$. It follows from (4.1b) below and (2.1b) that $d \rho_{2}$ is an invariant subgroup of $\rho_{1}$. We write $\bar{\rho}_{1}=\rho_{1} / d \rho_{2}$, and $\bar{x} \in \bar{\rho}_{1}$ will always denote the co-set containing a given element $x \in \rho_{1}$. The system $\rho$ and the operator $d$ shall satisfy the conditions:

(4.1) (a) $\rho_{1}$ is a free group,

(b) $\rho_{2}$ is a free crossed $\left(\rho_{1}, d_{2}\right)$-module,

(c) $\rho_{n}(n>2)$ is a free $\bar{\rho}_{1}$-module,

(d) $d_{r}$ is an operator homomorphism for each $r=2,3, \cdots$. That is to say, $d_{2}$ satisfies (2.1b), as required in the definition of a crossed module, and $d_{n} \bar{x}=\bar{x} d_{n}(n>2)$, for each operator $\bar{x} \in \bar{\rho}_{1}$.

We describe such a system as a homotopy system. If $\rho_{r}=0$ for $r>n \geqq 0$ but $\rho_{n} \neq 0$, in case $n>0$, we shall describe $\rho$ as $n$-dimensional and shall write $n=\operatorname{dim} \rho$ (if $r=1$ then $\rho_{r}=0$ means $\rho_{1}=1$ ).

For the sake of uniformity it will be convenient to let $\rho_{1}$ operate on $\rho_{n}(n>2)$ according to the rule $x a=\bar{x} a$, where $a \in \rho_{n}, x \in \rho_{1}$. Also, in order to include the case $n=1$ in such formulae as (4.3) below, we shall sometimes think of $\rho_{1}$ as an additive group, which operates on itself according to the rule $x y=x+y-x$. With these conventions (4.1d) reduces to $d x=x d$. When considered as a group of operators $\rho_{1}$ will always be multiplicative.

Let $\rho=\left\{\rho_{n}\right\}$ and $\rho^{\prime}=\left\{\rho_{n}^{\prime}\right\}$ be two homotopy systems and let $f_{1}: \rho_{1} \rightarrow \rho_{1}^{\prime}$ be a homomorphism such that $f_{1} d_{2} \rho_{2} \subset d_{2} \rho_{2}^{\prime}$, where $d$ denotes the boundary operator in both $\rho$ and $\rho^{\prime}$. Then $f_{1}$ induces a homomorphism, $\bar{f}_{1}: \bar{\rho}_{1} \rightarrow \bar{\rho}_{1}^{\prime}$, which is given by $\bar{f}_{1} \bar{x}=\overline{f_{1} x}$. Let $f_{n}: \rho_{n} \rightarrow \rho_{n}^{\prime}$ $(n \geqq 2)$ be an operator homomorphism, which is associated with $f_{1}$ if $n=2$ and with $\bar{f}_{1}$ if $n>2$. Then, with the convention explained in the preceding paragraph, we may describe $f_{n}$ as associated with $f_{1}$ for any $n \geqq 2$. Notice that $f_{1} d_{2} \rho_{2} \subset d_{2} \rho_{2}^{\prime}$ if there is a homomorphism, $f_{2}: \rho_{2} \rightarrow \rho_{2}^{\prime}$, such that $f_{1} d_{2}=d_{2} f_{2}$.

By a homomorphism, $f: \rho \rightarrow \rho^{\prime}$, we shall mean a family of homo-

\footnotetext{
${ }^{8} d_{3} \bar{x}=\bar{x} d_{3}$ has a meaning since $d_{3} \rho_{3} \in d_{2}^{-1}(1)$.
} 
morphisms, $f_{n}: \rho_{n} \rightarrow \rho_{n}^{\prime}(n \geqq 1)$, such that

(a) $f d=d f$ (i.e. $f_{n-1} d_{n}=d_{n} f_{n}$ for each $n \geqq 2$ ),

(b) $f_{n}$ is an operator homomorphism associated with $f_{1}$ if $n \geqq 2$.

We shall describe $f$ as an isomorphism if, and only if, $f_{n}$ is an isomorphism for each $n=1,2, \cdots$. We shall describe $\rho$ as isomorphic to $\rho^{\prime}$, and shall write $f: \rho \approx \rho^{\prime}$, if, and only if, there is an isomorphism $f: \rho \rightarrow \rho^{\prime}$. It is obvious that the resultant of homomorphisms (isomorphisms), $f: \rho \rightarrow \rho^{\prime}$ and $f^{\prime}: \rho^{\prime} \rightarrow \rho^{\prime \prime}$, is a homomorphism (isomorphism), $f^{\prime} f: \rho \rightarrow \rho^{\prime \prime}$; also that the inverse of an isomorphism is itself an isomorphism.

We now define a relation of equivalence, $\rho \equiv \rho^{\prime}$, which is the algebraic analogue of "being of the same homotopy type" between complexes. By a deformation operator, $\xi: \rho \rightarrow \rho^{\prime}$, associated with a homomorphism, $f_{1}:\left(\rho_{1}, d \rho_{2}\right) \rightarrow\left(\rho_{1}^{\prime}, d \rho_{2}^{\prime}\right)$, we shall mean a family of maps, $\xi_{n}: \rho_{\eta_{n}-1} \rightarrow \rho_{n}(n \geqq 2)$, such that:

(a) $\xi_{2}: \rho_{1} \rightarrow \rho_{2}^{\prime}$ is a crossed homomorphism associated with $f_{1}$,

(b) $\xi_{n}: \rho_{n_{i}-1} \rightarrow \rho_{n}^{\prime}(n>2)$ is an operator homomorphism associated with $f_{1}$.

We shall describe a homomorphism, $f: \rho \rightarrow \rho^{\prime}$, as equivalent to a homomorphism, $g: \rho \rightarrow \rho^{\prime}$, and shall write $f \simeq g$, if, and only if, there is a deformation operator, $\xi: \rho \rightarrow \rho^{\prime}$, associated with $f_{1}$, and an element $w^{\prime} \in \rho_{1}^{\prime}$, such that

$$
w^{\prime} g_{n}-f_{n}=d_{n+1} \xi_{n+1}+\xi_{n} d_{n} \quad(n \geqq 1),
$$

where $\xi_{1} d_{1}=0$. When $n=1$ this is another way of writing

$$
w^{\prime}\left(g_{1} x\right) w^{\prime-1}\left(f_{1} x\right)^{-1}=d_{2} \xi_{2} x \quad\left(x \in \rho_{1}\right) .
$$

We shall describe a homomorphism, $f: \rho \rightarrow \rho^{\prime}$, as an equivalence, if, and only if, there is a homomorphism, $f^{\prime}: \rho^{\prime} \rightarrow \rho$, such that $f^{\prime} f \simeq 1$, $f f^{\prime} \simeq 1$, where 1 denotes the identical isomorphism both in $\rho$ and in $\rho^{\prime}$. We shall describe $\rho$ as equivalent to $\rho^{\prime}$, and shall write $\rho \equiv \rho^{\prime}$, if, and only if, there is an equivalence, $f: \rho \rightarrow \rho^{\prime}$.

THEOREM 1 . The relations, $\simeq$, between homomorphisms $\rho \rightarrow \rho^{\prime}$, and $\equiv$, between homotopy systems, are equivalence relations.

It is not difficult to prove this directly. However we shall deduce it from theorems proved in $\S \S 10,11$ below.

Let $f \simeq g: \rho \rightarrow \rho^{\prime}$. Then it follows from (4.4) that

$$
\bar{f}_{1}=\alpha \bar{g}_{1}: \bar{\rho}_{1} \rightarrow \bar{\rho}^{\prime},
$$

where $\alpha$ is an inner automorphism of $\bar{\rho}_{1}^{\prime}$. Let $f: \rho \rightarrow \rho^{\prime}$ be an equivalence and let $f^{\prime}: \rho^{\prime} \rightarrow \rho$ be such that $f^{\prime} f \simeq 1, f f^{\prime} \simeq 1$. Then $\bar{f}_{1}^{\prime} \bar{f}_{1}=\alpha, \bar{f}_{1} \bar{f}_{1}^{\prime}=\alpha^{\prime}$, 
where $\alpha, \alpha^{\prime}$ are inner automorphisms of $\bar{\rho}_{1}, \bar{\rho}_{1}^{\prime}$. Hence it follows that $\bar{f}_{1}$ is an isomorphism.

5. The homotopy system of a complex. By a complex we shall mean a connected, CW-complex, as defined in (I), which may be finite or infinite. The characteristic maps of the cells in a complex will now be maps $I^{n} \rightarrow \bar{e}^{n}$ (not $\sigma^{n} \rightarrow \bar{e}^{n}$ ), where $I^{n}$ is the $n$-cube in Hilbert space, which is given by $0 \leqq t_{1}, \cdots, t_{n} \leqq 1, t_{i}=0$ if $i>n \geqq 0$, with $I^{0}$ $=(0,0, \cdots)$.

Our conventions concerning homotopy groups are as follows. We treat $I^{n}$ as $I \times I^{n-1}(n \geqq 1)$ and define

$$
I_{1}^{n-1}=1 \times I^{n-1}, \quad E^{n-1}=\partial I^{n}-\left(I_{1}^{n-1}-\partial I_{1}^{n-1}\right) .
$$

Thus $I_{1}^{n-1}$ is the face of $I^{n}$ in which $t_{1}=1$. Let $k: I^{n-1} \rightarrow I_{1}^{n-1}$ be given by $k p=(1, p)\left(p \in I^{n-1}\right)$. Let spaces $P, P_{0} \subset P$ and a point $p_{0} \in P$ be given. Then an element $a \in \pi_{r}\left(P, P_{0}, p_{0}\right)(r \geqq 2)$ shall be a homotopy class of maps of the form

$$
\theta:\left(I^{r}, \partial I^{r}, E^{r-1}\right) \rightarrow\left(P, P_{0}, p_{0}\right) .
$$

Let

$$
\beta_{r}: \pi_{r}\left(P, P_{0}, p_{0}\right) \rightarrow \pi_{r-1}\left(P_{0}, p_{0}, p_{0}\right)=\pi_{r-1}\left(P_{0}, p_{0}\right)
$$

be the boundary homomorphism, which we define as follows. If $\theta \in a$, then $\beta_{r} a$ shall be the element defined by the map

$$
\phi k: I^{r-1} \rightarrow P_{0}
$$

where $\phi=\theta \mid I_{1}^{r-1}: I_{1}^{r-1} \rightarrow P_{0}$.

Let

$$
\delta_{t}:\left(I^{r}, \partial I^{r}, I^{0}\right) \rightarrow\left(I^{r}, \partial I^{r}, I^{0}\right)
$$

be a fixed homotopy such that $\delta_{0}=1, \delta_{1} E^{r-1}=I^{0}$. Then we say that a map

$$
\phi:\left(I^{r}, \partial I^{r}, I^{0}\right) \rightarrow\left(P, P_{0}, p_{0}\right)
$$

represents the element of $\pi_{r}\left(P, P_{0}, p_{0}\right)$, which is defined by the map $\phi \delta_{1}: I^{r} \rightarrow P$. Similarly a map

$$
\psi:\left(\partial I^{r}, I^{0}\right) \rightarrow\left(P_{0}, p_{0}\right)
$$

will be said to represent the element of $\pi_{r-1}\left(P_{0}, p_{0}\right)$, which is defined by the map

$$
\left(\psi \delta_{1} \mid I_{1}^{r-1}\right) k: I^{r-1} \rightarrow P_{0}
$$


A path

$$
\lambda:(I, 0,1) \rightarrow\left(P_{0}, p_{0}, p_{0}^{\prime}\right)
$$

determines an isomorphism

$$
\bar{\lambda}: \pi_{r}\left(P, P_{0}, p_{0}^{\prime}\right) \rightarrow \pi_{r}\left(P, P_{0}, p_{0}\right),
$$

and also its inverse, $\bar{\lambda}^{-1}$. Let

$$
\theta^{\prime}:\left(I^{r}, \partial I^{r}, I^{0}\right) \rightarrow\left(P, P_{0}, p_{0}^{\prime}\right)
$$

be a map which represents an element $a^{\prime} \in \pi_{r}\left(P, P_{0}, p_{0}^{\prime}\right)$. Then we describe $\bar{\lambda} a^{\prime}$ as the element which is represented by the map $\theta^{\prime}$, joined to $p_{0}$ by the path $\lambda$. If $p_{0}^{\prime}=p_{0}$ we write $\bar{\lambda} a^{\prime}=x a^{\prime}$, where $x \in \pi_{1}\left(P_{0}, p_{0}\right)$ $=\pi_{1}^{0}$, say, is the element represented by the path $\lambda$. When $x a^{\prime}$ is thus defined, $\pi_{r}\left(P, P_{0}, p_{0}\right)$ is a $\pi_{1}^{0}$-module, if $r>2$, and a crossed

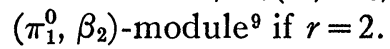

Let spaces $Q, Q_{0} \subset Q$ and a point $q_{0} \in Q_{0}$ be given. Then a map

$$
\phi:\left(P, P_{0}, p_{0}\right) \rightarrow\left(Q, Q_{0}, q_{0}\right)
$$

induces a homomorphism

$$
f: \pi_{r}\left(P, P_{0}, p_{0}\right) \rightarrow \pi_{r}\left(Q, Q_{0}, q_{0}\right) .
$$

Let $a \in \pi_{r}\left(P, P_{0}, p_{0}\right)$ be represented by the map (5.1), joined to $p_{0}$ by the path $\lambda$. Then it is easily verified that $f a$ is represented by the map $\phi \theta^{\prime}$, joined to $q_{0}$ by the path $\phi \lambda$. Taking $p_{0}^{\prime}=p_{0}$ it follows that $f$ is an operator homomorphism associated with the homomorphism, $\pi_{1}\left(P_{0}, p_{0}\right) \rightarrow \pi_{1}\left(Q_{0}, q_{0}\right)$, which is induced ${ }^{10}$ by $\phi$.

Similar remarks apply to $\pi_{r-1}\left(P_{0}, p_{0}\right)$ and $\pi_{r-1}\left(P_{0}, p_{0}^{\prime}\right)$, related to each other by the path $\lambda$.

Let $K$ be a given complex and let a 0 -cell, $e^{0} \in K^{0}$, be taken as base-point for all groups $\pi_{n}\left(K^{n}, K^{n-1}\right), \pi_{n}(K)$, etc. Let

$$
\begin{aligned}
\rho_{r} & =\rho_{r}(K)=\pi_{r}\left(K^{r}, K^{r-1}\right) \\
\rho_{1} & =\rho_{1}(K)=\pi_{1}\left(K^{1}\right) .
\end{aligned}
$$

Let

$$
j_{n}: \pi_{n}\left(K^{n}\right) \rightarrow \rho_{n} \quad\left(n \geqq 1 ; j_{1}=1\right)
$$

be the homomorphism induced by the identical map $\left(K^{n}, e^{0}, e^{0}\right)$ $\rightarrow\left(K^{n}, K^{n-1}, e^{0}\right)$ and let 11

${ }^{9}$ See $[12$, p. 486$]$, which may be taken as a general reference for the elementary propositions stated here.

${ }^{10}$ I.e. induced by $\phi \mid P_{0}: P_{0} \rightarrow Q_{0}$.

${ }^{11}$ Cf. [8, p. 429], with $X_{i}=K^{i}(i>0)$. 


$$
d_{r}=j_{r-1} \beta_{r}: \rho_{r} \rightarrow \rho_{r-1} .
$$

Then $d_{2}=\beta_{2}$. Since $\beta_{r} j_{r}=0$ we have $d_{r} d_{r+1}=0$. If $r \geqq 3$ the group $\rho_{r}$ is a free $\pi_{1}\left(K^{r-1}\right)$-module ${ }^{12}$ and we identify ${ }^{13} \pi_{1}\left(K^{n}\right)(n>1)$, as well as $\pi_{1}(K)$, with $\bar{\rho}_{1}=\rho_{1} / d_{2} \rho_{2}$. Also $\rho_{2}$ is a free $\operatorname{crossed}^{12}\left(\rho_{1}, d_{2}\right)$-module and $d_{r}$ is an operator homomorphism in the sense of $(4.1 \mathrm{~d})$. Therefore $\left\{\rho_{n}\right\}$ is a homotopy system, which we call the homotopy system, $\rho(K)$, of $K$.

Let $\left\{e_{i}^{n}\right\}(n \geqq 2)$ be the $n$-cells in $K$ and let $a_{i} \in \rho_{n}$ be the element represented by a characteristic map for $e_{i}^{n}$, joined to $e^{0}$ by a path in $K^{n-1}$. Then $\left\{a_{i}\right\}$ is a basis, which we describe as a natural basis, for $\rho_{n}$. Let $T \subset K^{1}$ be a tree (cf. [4, p. 322]) which is a sub-complex containing $K^{0}$, and let $\left\{e_{i}^{1}\right\}$ be the 1-cells in $K^{1}-T$. Let $g_{i}: I \rightarrow \tilde{e}_{i}^{1}$ be a characteristic map for $e_{i}^{1}$ and let $x_{i} \in \rho_{1}$ be the element represented by a path in $T$, which leads from $e^{0}$ to $g_{i}(0)$, followed by $g_{i}$, followed by a path in $T$, which leads from $g_{i}(1)$ back to $e^{0}$. Then $\left\{x_{i}\right\}$ is a basis for $\rho_{1}$.

A (cellular) map, ${ }^{14} \phi: K \rightarrow L$, in a complex $L$, with base point $e^{\prime 0}=\phi e^{0}$, obviously induces a homomorphism, $f: \rho(K) \rightarrow \rho(L)$. If $g: \rho(L) \rightarrow \rho(M)$ is the homomorphism induced by a map, $\psi: L \rightarrow M$, into a complex $M$, then $g f: \rho(K) \rightarrow \rho(M)$ is obviously the homomorphism induced by $\psi \phi: K \rightarrow M$. If $f: \rho(K) \rightarrow \rho(L)$ is a given homomorphism and if $\phi: K \rightarrow L$ is a map which induces it we shall describe $\phi$ as a realization of $f$.

Let $f: \rho(K) \rightarrow \rho(L)$ be a given homomorphism and let $K_{0} \subset K$ be a connected sub-complex, which contains $e^{0}$. We shall describe a map, $\phi_{0}: K_{0} \rightarrow L$, as a partial realization of $f$ if, and only if,

$$
f^{0}=f i: \rho\left(K_{0}\right) \rightarrow \rho(L),
$$

where $f^{0}$ and $i: \rho\left(K_{0}\right) \rightarrow \rho(K)$ are the homomorphisms induced by $\phi_{0}$ and by the identical map $K_{0} \rightarrow K$. We shall call $i$ the injection homomorphism.

Let $K_{1} \subset K$ be a (connected) sub-complex, which contains ${ }^{15} K^{n-1}$

12 See $\$ 16$ below.

${ }^{13}$ I.e. we use $\bar{\rho}_{1}$ and $\bar{x} \in \bar{\rho}_{1}$ to denote $\pi_{1}(K)$ and $i \bar{x} \in \pi_{1}(K)$, etc., where $i: \bar{\rho}_{1} \rightarrow \pi_{1}(K)$ is the ismorphism in which corresponding elements are represented by the same map $I \rightarrow K^{1}$.

${ }^{14}$ It is to be understood that all our maps and homotopies are cellular (see $L$ in $\$ 5$ of (I)) and, except when the contrary is stated, that a given map, $K \rightarrow L$, carries the base point of $K$ into the base point of $L$.

${ }^{15}$ The condition $K^{n-1} \subset K_{1}$ is not necessary to Lemma 4 below but it helps to simplify the notation. 
$(n \geqq 2)$. Let $K_{1}=K_{0} \cup\left\{e_{\sigma}^{n}\right\}$, where $\left\{e_{\sigma}^{n}\right\}=K_{1}-K_{0}$ is a set of $n$-cells, each of which is a principal cell (i.e. a cell which is an open sub-set) of $K_{1}$. Let $\phi_{0}: K_{0} \rightarrow L$ be a partial realization of a given homomorphism, $f: \rho(K) \rightarrow \rho(L)^{\circ}$, and let $g: \pi_{n-1}\left(K^{n-1}\right) \rightarrow \pi_{n-1}\left(L^{n-1}\right)$ be the homomorphism which is induced by $\phi_{0}$. Let $a_{\sigma} \in \rho_{n}(K)$ be the element which is represented by a characteristic map, $h_{\sigma}: I^{n} \rightarrow \bar{e}_{\sigma}^{n}$, for $e_{\sigma}^{n}$, joined to $e^{0}$ by a path

$$
\lambda_{\sigma}:(I, 0,1) \rightarrow\left(K^{n-1}, e^{0}, p_{\sigma}\right) \quad\left(p_{\sigma}=h_{\sigma} I^{0}\right) .
$$

Lemma 4. If ${ }^{16} \beta f a_{\sigma}=g \beta a_{\sigma}$, for each $\sigma$, then $\phi_{0}$ can be extended to a partial realization, $\phi_{1}: K_{1} \rightarrow L$, of $f$.

This is essentially the same as Lemma 7 in $\$ 9$ of [6], except that we must now pay attention to base points. Let $\beta f a_{\sigma}=g \beta a_{\sigma}$, let $q_{\sigma}=\phi_{0} p_{\sigma}$ and let

$$
\mu_{\sigma}=\phi_{0} \lambda_{\sigma}:(I, 0,1) \rightarrow\left(L^{n-1}, e^{\prime}, q_{\sigma}\right) .
$$

Let $\mu_{\sigma}^{\prime}: \rho_{n}(L) \rightarrow \pi_{n}\left(L^{n}, L^{n-1}, q_{\sigma}\right)$ be the isomorphism determined by the path $\mu_{\sigma}$ and let

$$
k_{\sigma}:\left(I^{n}, \partial I^{n}, I^{0}\right) \rightarrow\left(L^{n}, L^{n-1}, q_{\sigma}\right)
$$

be a map which represents the element $\mu_{\sigma}^{\prime} f a_{\sigma}$. Then $k_{\sigma} \mid \partial I^{n}$ and $\phi_{0} h_{\sigma} \mid \partial I^{n}$ both represent the image of $\beta f a_{\sigma}=g \beta a_{\sigma}$ in the isomorphism,

$$
\pi_{n-1}\left(L^{n-1}, e^{\prime 0}\right) \rightarrow \pi_{n-1}\left(L^{n-1}, q_{\sigma}\right),
$$

which is determined by the path $\mu_{\sigma}$. Therefore it follows from the proof of Lemma 7 in [6], applied to each cell $e_{\sigma}^{n}$, that $\phi_{0}$ can be extended to a map, $\phi_{1}: K_{1} \rightarrow L$, such that $f a_{\sigma}$ is represented by the map $\phi_{1} h_{\sigma}: I^{n} \rightarrow L^{n}$, joined to $e^{\prime 0}$ by the path $\mu_{\sigma}$.

Let $f^{r}: \rho\left(K_{r}\right) \rightarrow \rho(L)(r=0,1)$ be the homomorphism induced by $\phi_{r}$ and let $a_{\sigma}^{1} \in \rho_{n}\left(K_{1}\right)$ be the element represented by $h_{\sigma}$, joined to $e^{0}$ by the path $\lambda_{\sigma}$. Then $a_{\sigma}=i^{1} a_{\sigma}^{1}$, where $i^{1}$ is the injection $i^{1}: \rho\left(K_{1}\right) \rightarrow \rho(K)$, and $f^{1} a_{\sigma}^{1}=f a_{\sigma}=f i^{1} a_{\sigma}^{1}$. Let $i$ and $i^{0}$ be the injections $i: \rho\left(K_{0}\right) \rightarrow \rho\left(K_{1}\right)$ and $i^{0}=i^{1} i: \rho\left(K_{0}\right) \rightarrow \rho(K)$. Since $\phi_{1} \mid K_{0}=\phi_{0}$ it is obvious that $f^{1} i=f^{0}$. Also $f^{0}=f i^{0}=f i^{1} i$, since $\phi_{0}$ is a partial realization of $f$. Therefore $f^{1} i a^{0}$ $=f i^{1} i a^{0}$ if $a^{0} \in \rho_{n}\left(K_{0}\right)$. If $\left\{a_{j}^{0}\right\}$ is a natural basis for $\rho_{n}\left(K_{0}\right)$ then $\left\{i a_{j}^{0}, a_{\sigma}^{1}\right\}$ is a natural basis for $\rho_{n}\left(K_{1}\right)$. Since $f^{1} b=f i^{1} b$ if $b=i a_{j}^{0}$ or $a_{\sigma}^{1}$ and also (obviously) if $b \in \rho_{r}\left(K_{1}\right)$ with $r \neq n$ it follows that $f^{1}=f i^{1}$. Therefore $\phi_{1}$ is a partial realization of $f$ and the lemma is proved.

In general a homomorphism $f: \rho(K) \rightarrow \rho(L)$ cannot be realized by a map $K \rightarrow L$. For example a complex projective plane and the union of a 2-sphere and a 4-sphere with a single common point may be covered

${ }^{16}$ We shall sometimes write $\beta, f$, etc. for $\beta_{n}, f_{n}$, etc. 
by complexes, $K$ and $L$, each of which consists of a 0 -cell, a 2-cell and a 4 -cell. Then $\rho_{n}(K) \approx \rho_{n}(L)$, both groups being cyclic infinite if $n=2$ or 4 and zero otherwise. By considering the cohomology rings, or the groups $\pi_{3}(K), \pi_{3}(L)$, one sees that an isomorphism $\rho(K) \rightarrow \rho(L)$ cannot be realized by a map $K \rightarrow L$.

6. Realizability. We shall describe a complex, $K$, as a (geometrical) realization of a given homotopy system, $\rho$, if, and only if, $\rho \approx \rho(K)$. In $\$ 15$ below we given an example of a homotopy system which has no geometrical realization. Thus it is not always possible to realize either a homormophism $\rho(K) \rightarrow \rho(L)$ by a map $K \rightarrow L$ or a homotopy system $\rho$ by a complex $K$. In this section we prove three theorems which state sufficient conditions for both kinds of realizability to be possible.

Theorem 2. A given homotopy system, $\rho$, has a geometrical realization if $\operatorname{dim} \leqq 4$.

Let $n=\operatorname{dim} \rho$ and first let $n=1$. Then $\rho$ is obviously' realized by a complex $K=e^{0} \cup\left\{e_{i}^{1}\right\}$, where $\left\{e_{i}^{1}\right\}$ is a set of 1-cells in (1-1) correspondence with a basis, $\left\{x_{i}\right\}$, for $\rho_{1}$.

We proceed by induction on $n$. Let $\rho^{r}$ be the homotopy system

$$
\rho^{r}=\left(\rho_{1}, \cdots, \rho_{r}, 0,0, \cdots\right),
$$

$d_{s}$ being the same in $\rho^{r}$ as in $\rho$ if $2 \leqq s \leqq r$. Assume that $\rho^{n-1}$ is realized by a complex $K^{n-1}(1<n \leqq 4)$ and let $f^{n-1}: \rho^{n-1} \approx \rho\left(K^{n-1}\right)$. Let $\left\{a_{i}\right\}$ be a basis for $\rho_{n}$. If $n>2$ we have $d_{n-1} d_{n}=0$, whence

$$
d_{n-1} f_{n-1}^{n-1} d_{n} a_{i}=f_{n-2}^{n-1} d_{n-1} d_{n} a_{i}=0,
$$

or $f_{n-1}^{n-1} d_{n} a_{i} \in d_{n-1}^{-1}(0)$. Since $n-1 \leqq 3$

$$
j_{n-1}: \pi_{n-1}\left(K^{n-1}\right) \rightarrow \rho_{n-1}\left(K^{n-1}\right)
$$

is onto ${ }^{17} d_{n-1}^{-1}(0)$. Also $f_{1}^{1} d_{2} a_{i} \in \pi_{1}\left(K^{1}\right)$ if $n=2$. Therefore there is an element $b_{i} \in \pi_{n-1}\left(K^{n-1}\right)$, such that

$$
f_{n-1}^{n-1} d_{n} a_{i}=j_{n-1} b_{i} \quad(n \geqq 2) .
$$

Let $g_{i}:\left(\partial I^{n}, I^{0}\right) \rightarrow\left(K^{n-1}, e^{0}\right)$ be a map which represents $b_{i}$. Let $\left\{E_{i}^{n}\right\}$ be a set of $n$-elements, which are disjoint from each other and from $K^{n-1}$ and let $h_{i}: \partial E_{i}^{n} \rightarrow \partial I^{n}$ be a homeomorphism. We attach $e_{i}^{n}=E_{i}^{n}-\partial E_{i}^{n}$ to $K^{n-1}$ by means of the map $g_{i} h_{i}: \partial E_{i}^{n} \rightarrow K^{n-1}$. The result is a complex, $K^{n}=K^{n-1} \cup\left\{e_{i}^{n}\right\}$, in which $e_{i}^{n}$ has a characteristic map,

${ }^{17}$ See Theorem 7 in $\$ 3$ of $(I)$. 
$g_{i}^{\prime}: I^{n} \rightarrow \vec{e}_{i}^{n}$, such that $g_{i}^{\prime} \mid \partial I^{n}=g_{i}$. Let $a_{i}^{\prime} \in \rho_{n}\left(K^{n}\right)$ be the element represented by $g_{i}^{\prime}$. Then $\beta_{n} a_{i}^{\prime}=b_{i}$, whence

$$
d_{n} a_{i}^{\prime}=j_{n-1} b_{i}=f_{n-1}^{n-1} d_{n} a_{i} .
$$

Hence, and from Lemma 2 if $n=2$, an operator homomorphism, $f_{n}: \rho_{n} \rightarrow \rho_{n}\left(K^{n}\right)$, which is obviously an isomorphism, is defined by $f_{n} a_{i}=a_{i}^{\prime}$. Moreover it also follows from (6.1) that $d_{n} f_{n}=f_{n-1}^{n-1} d_{n}$. Therefore we have defined an isomorphism $f: \rho \rightarrow \rho\left(K^{n}\right)$, where $f_{r}=f_{r}^{n-1}$ if $r<n$, and the theorem follows by induction on $n$.

THEOREM 3. A homotopy system, $\rho$, has a geometrical realization if $\bar{\rho}_{1}=1$.

Let $\bar{\rho}_{1}=1$. Then $\rho_{r}(r \geqq 3)$ and hence any sub-group of $\rho_{r}$ is a free Abelian group. ${ }^{18}$ Therefore $d_{n} \rho_{n}$ is a free Abelian group $(n \geqq 4)$. Let $\left\{b_{i}\right\}$ be a basis for $d_{n} \rho_{n}$, let $c_{i} \in d_{n}^{-1} b_{i}$ and let $C_{n} \subset \rho_{n}$ be the sub-group generated by $\left\{c_{i}\right\}$. A relation between the elements $\left\{c_{i}\right\}$ would imply the corresponding relation between $\left\{b_{i}\right\}$, whence $\left\{c_{i}\right\}$ is a basis for $C_{n}$. Therefore, as in elementary homology theory, $\rho_{n}$ is the direct sum $\rho_{n}=Z_{n}+C_{n}$, where $Z_{n}=d_{n}^{-1}(0)$, and $d_{n} \mid C_{n}$ is an isomorphism onto $d_{n} \rho_{n}$. Let $\left\{z_{\lambda}^{n}\right\}$ be a basis for $Z_{n}$.

By Theorem $2, \rho^{3}$ has a realization $K^{3}$, where $\rho^{r}$ means the same as before. Let $n \geqq 4$ and assume that there is a complex, $K^{n-1}$, such that

$$
f^{n-1}: \rho^{n-1} \approx \rho\left(K^{n-1}\right)
$$

and

$$
j_{n-1}: \pi_{n-1}\left(K^{n-1}\right) \rightarrow \rho_{n-1}\left(K^{n-1}\right)
$$

is onto $d_{n-1}^{-1}(0)$. Then the proof of Theorem 2 shows that there is a complex,

$$
K^{n}=K^{n-1} \cup\left\{e^{n}\right\},
$$

such that $f^{n}: \rho^{n} \approx \rho\left(K^{n}\right)$ and $f^{n} \mid \rho^{n-1}=f^{n-1}$. Moreover we may suppose that $\partial e_{\lambda}^{n}=e^{0} \in K^{0}$, for each value of $\lambda$, where $e_{\lambda}^{n}$ is the cell which corresponds to $z_{\lambda}^{n} \in Z_{n \text {. }}$ Let this be so and let $P^{n}$ be the union of the $n$-spheres $\bar{e}_{\lambda}^{n}$. Then any element in $Z_{n}$ is represented by a map, $\left(I^{n}, \partial I^{n}\right) \rightarrow\left(P^{n}, e^{0}\right)$, which also represents an element in $\pi_{n}\left(K^{n}\right)$. Therefore $j_{n}$ is onto $Z_{n}$. Therefore complexes $K^{3} \subset K^{4} \subset \cdots$ and iso-

\footnotetext{
${ }^{18} \mathrm{We}$ assume here that $\rho_{r}$ has a well-ordered basis. Then this assertion follows by transfinite induction in much the same way as when the basis is finite. I do not know if this can be proved without the axiom of choice.
} 
morphisms $f^{n}: \rho^{n} \approx \rho\left(K^{n}\right)$, such that $f^{n} \mid \rho^{n-1}=f^{n-1}$, are defined inductively for every value of $n \geqq 3$. Let

$$
K=\bigcup_{n} K^{n}
$$

with the topology defined by the condition that $X \subset K$ is closed if, and only if, $X \cap K^{n}$ is a closed sub-set of $K^{n}$, for each $n=3,4, \cdots$. Then $K$ is a complex, whose $n$-section is $K^{n}$ and $f: \rho \rightarrow \rho(K)$ is obviously an isomorphism, where $f \mid \rho^{n}=f^{n}$. This proves the theorem.

Let $K, L$ be given complexes, let $f: \rho(K) \rightarrow \rho(L)$ be a given homomorphism and let $\phi_{0}: K_{0} \rightarrow L$ be a partial realization of $f$, where $K_{0} \subset K$ is a connected sub-complex, which contains $e^{0}$.

Theorem 4. If $K_{0} \cup K^{m+1}=K$ and if $L$ is a $J_{m}$-complex, then $\phi_{0}$ can be extended to a complete realization, ${ }^{19} \phi: K \rightarrow L$, of $f$.

Let a basis for $\rho_{1}(K)$ be defined, as in $\$ 5$, in terms of a tree, $T \subset K^{1}$, such that $K^{0} \subset T$. We assume that $T$ consists of a tree, ${ }^{20} T_{0} \subset K_{0}$, which contains $K_{0}^{0}$, together with a set of trees, $\left\{T_{i}\right\}$, such that $T_{i} \cap K_{0}$ is a single 0 -cell $e_{i}^{0}$. We map $T_{i}$ on $\phi_{0} e_{i}^{0}$, thus extending $\phi_{0}$ throughout $K_{0} \cup T$. It now follows from a simplified version of Lemma 4 that $\phi_{0}$ can be extended to a partial realization, $\phi_{1}: K_{0} \cup K^{1}$ $\rightarrow L$, of $f$.

Let $K_{r}=K_{0} \cup K^{r}(r \geqq 1)$ and assume that $\phi_{0}$ has been extended to a partial realization, $\phi_{n-1}: K_{n-1} \rightarrow L$, of $f$, where $2 \leqq n \leqq m+1$. Let $g: \pi_{n-1}\left(K^{n-1}\right) \rightarrow \pi_{n-1}\left(L^{n-1}\right)$ be the homomorphism induced by $\phi_{n-1}$. Then it is easily verified that

$$
f_{n-1} j_{n-1}=j_{n-1} g: \pi_{n-1}\left(K^{n-1}\right) \rightarrow \rho_{n-1}(L) .
$$

Therefore

$$
j_{n-1} \beta_{n} f_{n}=d_{n} f_{n}=f_{n-1} d_{n}=f_{n-1} j_{n-1} \beta_{n}=j_{n-1} g \beta_{n} .
$$

Let $L$ be a $J_{m}$-complex. Then $j_{n-1}^{-1}(0)=0$, since $n-1 \leqq m$. Therefore $\beta_{n} f_{n}=g \beta_{n}$ and it follows from Lemma 4 that $\phi_{n-1}$ can be extended to a partial realization, $\phi_{n}: K_{n} \rightarrow L$, of $f$. The theorem now follows by induction on $n$.

7. Homotopy and equivalence. We state three theorems, which will be proved in $\$ \$ 13,14$ below. Let $K, L$ be given complexes and let $f, f^{\prime}: \rho(K) \rightarrow \rho(L)$ be the homomorphisms induced by maps $\phi, \phi^{\prime}: K \rightarrow L$.

\footnotetext{
${ }^{19} K_{0}$ may consist of the single 0 -cell $e^{0}$, in which case the theorem asserts that $f$ has a realization if $\operatorname{dim} K \leqq m+1 . J_{m}$-complexes are defined in (I), $\S 3$.

${ }^{20}$ In constructing $T$ we can start with $T_{0}$.
} 
THEOREM 5. If $\phi \simeq \phi^{\prime}$ then $f \simeq f^{\prime}$.

Theorem 6. A map $\phi: K \rightarrow L$ is a homotopy equivalence if, and only if, the induced homomorphism, $f: \rho(K) \rightarrow \rho(L)$, is an equivalence.

As a corollary to Theorems 4 and 6 we have:

Corollary. If $\operatorname{dim} K \leqq m+1$ and if $L$ is a $J_{m}$-complex, then $K \equiv L$ if, and only if, $\rho(K) \equiv \rho(L)$. More precisely, an equivalence, $\rho(K) \rightarrow \rho(L)$, can be realized by a homotopy equivalence, $K \rightarrow L$, and conversely.

Since every complex is a $J_{2}$-complex it follows from this and Theorem 2 that an equivalence class of 3-dimensional homotopy systems is an algebraic equivalent of the homotopy type of a 3-dimensional complex. Moreover, if $\operatorname{dim} \rho \leqq 3$ and if $K, L$ are any complexes such that $\rho(K) \equiv \rho(L) \equiv \rho$, then $K \equiv L$, even if $\operatorname{dim} K, \operatorname{dim} L>3$. For it follows from Theorem 2 that $\rho$ can be realized by a complex, $P^{3}$, and it follows from Theorem 4 that equivalences, $\rho\left(P^{3}\right) \rightarrow \rho(K)$, $\rho\left(P^{3}\right) \rightarrow \rho(L)$ can be realized by maps $P^{3} \rightarrow K, P^{3} \rightarrow L$, which are homotopy equivalences by Theorem 6 . Therefore $K \equiv P^{3} \equiv L$. In a later paper we shall prove that if $\rho \equiv \rho^{\prime}, \operatorname{dim} \rho, \operatorname{dim} \rho^{\prime}<\infty$, where $\rho, \rho^{\prime}$ are given homotopy systems, and if $\rho$ can be realized geometrically, so can $\rho^{\prime}$. Therefore, if an equivalence class of homotopy systems contains one, $\rho$, such that $\operatorname{dim} \rho \leqq 3$, then every homotopy system in the class can be realized geometrically and all the resulting complexes are of the same homotopy type.

By taking $K=S^{3}, L=S^{2}$ we see that the converse of Theorem 5, namely $f \simeq f^{\prime}$ implies $\phi \simeq \phi^{\prime}$, does not hold, even if $L$ is a $J_{m}$-complex and $\operatorname{dim} K \leqq m+1$. However we shall prove:

Theorem 7. If $L$ is a $J_{m}$-complex and $\operatorname{dim} K \leqq m$, then $f \simeq f^{\prime}$ implies $\phi \simeq \phi^{\prime}$.

It follows that the homotopy theory of 2-dimensional complexes, including the homotopy classification of mappings, is equivalent to the purely algebraic theory of free crossed modules, whose groups of operators are free groups.

8. Chain groups. With each homotopy system, $\rho=\left\{\rho_{n}\right\}$, which need not have a geometrical realization, we associate a system of chain groups, $C=\left\{C_{r}\right\}(r=0,1, \cdots)$. Each group $C_{r}$ shall be a free $\bar{\rho}_{1-}$ module and $C_{n}$ shall be operator isomorphic to $\rho_{n}$ if $n>2$ and to $\rho_{2}$ made Abelian if $n=2$. The group $C_{1}$ shall have a basis $\left\{c_{i}\right\}$ which is in a (1-1) correspondence, $x_{i} \rightarrow c_{i}$, with a basis $\left\{x_{i}\right\}$ for $\rho_{1}$. The group $C_{0}$ shall have a basis consisting of a single element $c^{0}$. The ele- 
ment $c^{0}$ is to be a "preferred" or special element in $C_{0}$. We allow $\rho_{1}$ to operate on $C_{n}$ in the same way as on $\rho_{n}(n>2)$.

The system $C$ shall be related to $\rho$ by a preferred family of maps,

$$
h_{n}: \rho_{n} \rightarrow C_{n} .
$$

The map $h_{n}$ shall be an operator isomorphism if $n>2$ and $h_{2}$ shall be an operator homomorphism (onto), whose kernel is the commutator sub-group of $\rho_{2}$. The map $h_{1}$ shall be the crossed homomorphism which is uniquely determined by $h x_{i}=c_{i}$, according to Lemma 3 .

We now define a boundary operator, $\partial$, which is a family of operator homomorphisms, $\partial_{n}: C_{n} \rightarrow C_{n-1}(n \geqq 1)$, such that $\partial_{n} \partial_{n+1}=0$. We shall define it in such a way that

$$
\begin{aligned}
\text { (a) } \partial_{1} h_{1} x & =(\bar{x}-1) c^{0} & & \left(x \in \rho_{1}\right), \\
\text { (b) } \partial_{n} h_{n} & =h_{n-1} d_{n} & & (n \geqq 2) .
\end{aligned}
$$

If $n>2$ we define $\partial_{n}=h_{n-1} d_{n} h_{n}^{-1}$. We postpone the definition of $\partial_{2}$ and define $\partial_{1}$ by $\partial_{1} c_{i}=\left(x_{i}-1\right) c^{0}$. Let $g: \rho_{1} \rightarrow C_{0}$ be the (principal) crossed homomorphism, which is given by $g x=(\bar{x}-1) c^{0}$. The map $\partial_{1} h_{1}: \rho_{1} \rightarrow c^{0}$ is the resultant of the crossed homomorphism $h_{1}$ and the operator homomorphism $\partial_{1}$. Therefore it is a crossed homomorphism. Moreover $g$ and $\partial_{1} h_{1}$ are both associated with the same homomorphism, $x \rightarrow \bar{x}$, of $\rho_{1}$ onto $\bar{\rho}_{1}$. Since $\partial_{1} h_{1} x_{i}=g x_{i}$, from the uniqueness part of Lemma $3 \partial_{1} h_{1}=g$, which is expressed by (8.1a).

THEOREM 8. The crossed homomorphism $h_{1}$ has the properties:

(a) $h_{1} \mid d_{2} \rho_{2}$ is a homomorphism onto $\partial_{1}^{-1}(0) \subset C_{1}$.

(b) $h_{1} x=h_{1} y\left(x, y \subset \rho_{1}\right)$ if, and only if, $x y^{-1} \in d_{2} h_{2}^{-1}(0)$. In particular $h_{1}^{-1}(0)=d_{2} h_{2}^{-1}(0)$.

(c) If $\partial_{1} c=(\bar{x}-1) c^{0}$, where $c \in C_{1}, \bar{x} \in \bar{\rho}_{1}$, then $c=h_{1} x^{\prime}$, for some $x^{\prime} \in \bar{x}$.

In order to prove this we introduce a complex, $K^{1}=e^{0} \cup\left\{e_{i}^{1}\right\}$, where $\left\{e_{i}^{1}\right\}$ is a set of 1 -cells which is in a (1-1) correspondence, $x_{i} \rightarrow e_{i}^{1}$, with $\left\{x_{i}\right\}$. We identify $x_{i}$ with the element, $x_{i} \in \pi_{1}\left(K^{1}\right)$, which is represented by a characteristic map for $e_{\imath}^{1}$. Let $\widetilde{K}^{1}$ be a covering complex of $K^{1}$, which is associated with the (invariant) sub-group $d_{2} \rho_{2} \subset \rho_{1}$, and let $p: \widetilde{K}^{1} \rightarrow K^{1}$ be the covering map. Then $p$ induces an isomorphism $p_{1}: \widetilde{\rho}_{1} \rightarrow d_{2} \rho_{2}$, where $\tilde{\rho}_{1}=\pi_{1}\left(\tilde{K}^{1}\right)$, with a 0 -cell $\tilde{e}^{0} \in p^{-1} e^{0}$ as base point. Following S. Eilenberg and N. E. Steenrod in [11] we take

$$
C_{0}^{\prime}=H_{0}\left(\tilde{K}^{0}\right), \quad C_{1}^{\prime}=H_{1}\left(\tilde{K}_{1}, \tilde{K}^{0}\right)
$$

as chain groups in $\widetilde{K}^{1}$. We denote the boundary homomorphism by $\partial^{\prime}: C_{1}^{\prime} \rightarrow C_{0}^{\prime}$. 
Let $\lambda:(I, 0,1) \rightarrow\left(K^{1}, e^{0}, e^{0}\right)$ be a map representing a given element, $x \in \rho_{1}$, and let $\tilde{\lambda}:(I, 0) \rightarrow\left(\tilde{K}^{1}, \tilde{e}^{0}\right)$ be the lifted map (i.e. $\left.\lambda=p \tilde{\lambda}\right)$. Let $h x \in C_{1}$ be the element represented by $\tilde{\lambda}$. Clearly $h x$ depends only on $x \in \rho_{1}$ and not on the choice of the representative map $\lambda$. Also

$$
\partial^{\prime} h x=(\bar{x}-1) c^{\prime 0},
$$

where $c^{\prime 0} \in C_{0}^{\prime}$ is the 0 -chain, which corresponds to $\widetilde{e}^{0}$, and $\bar{\rho}_{1}$ operates in the usual way on $C_{0}^{\prime}, C_{1}^{\prime}$. Also $\left\{h x_{i}\right\}$ is a basis for $C_{1}^{\prime}$. Let $y \in \rho_{1}$ be represented by a map $\mu:(I, 0,1) \rightarrow\left(K^{1}, e^{0}, e^{0}\right)$. Then $x y$ is represented by the circuit $\lambda$ followed by $\mu$ and $h(x y)$ by the path $\tilde{\lambda}$ followed by $\mu^{\prime}: I \rightarrow \widetilde{K}^{1}$, where $\mu=p \mu^{\prime}$ and $\mu^{\prime}(0)=\tilde{\lambda}(1)$. Therefore

$$
h(x y)=h x+\bar{x} h y .
$$

We identify $C_{0}, C_{1}$ with $C_{0}^{\prime}, C_{1}^{\prime}$ in such a way that $c_{i}=h x_{i}, c^{0}=c^{\prime 0}$. Then it follows from (8.2), (8.3) and the uniqueness part of Lemma 3 that $h=h_{1}$. Hence, and by (8.1a), $\partial^{\prime}=\partial_{1}$. Since $\bar{x}=1$ if $x \in d_{2} \rho_{2}$, it follows from (8.3) and (8.2) that $h_{1} \mid d_{2} \rho_{2}$ is a homomorphism into $\partial_{1}^{-1}(0)$. It follows from the geometrical definition of $h_{1}$ that $h_{1} \mid d_{2} \rho_{2}$ $=h^{\prime} p^{-1}$, where $h^{\prime}: \tilde{\rho}_{1} \rightarrow \partial_{1}^{-1}(0)$ is the homomorphism in which corresponding elements are represented by the same map. Therefore it follows from the well known relation between the fundamental group of a polyhedron and its first homology group that $h_{1} \mid d_{2} \rho_{2}$ is onto $\partial_{1}^{-1}(0)$, which establishes (a).

If $h_{1} x=0$, then $(\bar{x}-1) c^{0}=\partial_{1} h_{1} x=0$, whence $\bar{x}=1$ and $x \in d_{2} \rho_{2}$. Therefore, writing $G^{c}$ for the commutator sub-group of a given group, $G$, we have

$$
h_{1}^{-1}(0)=\left(h_{1} \mid d_{2} \rho_{2}\right)^{-1}(0)=p_{1}{h^{\prime}}^{-1}(0)=p_{1} \tilde{\rho}_{1}^{0}
$$

But $f\left(G^{c}\right)=(f G)^{c}$ if $f$ is any homomorphism of $G$ onto any other group. Therefore

$$
h_{1}^{-1}(0)=\left(p_{1} \tilde{\rho}_{1}\right)^{c}=\left(d_{2} \rho_{2}\right)^{c}=d_{2} \rho_{2}^{0}=d_{2} h_{2}^{-1}(0) .
$$

Let $x y^{-1}=z \in d_{2} h_{2}^{-1}(0)$. Then $h_{1} z=0$ and $\bar{z}=1$, since $z \in d_{2} \rho_{2}$. Therefore

$$
h_{1} x=h_{1}(z y)=h_{1} y .
$$

Conversely, let $h_{1} x=h_{1} y$. Then

$$
(\bar{x}-1) c^{0}=\partial_{1} h_{1} x=\partial_{1} h_{1} y=(\bar{y}-1) c^{0},
$$

whence $\bar{x}=\bar{y}$. Therefore

$$
h_{1}\left(x y^{-1}\right)=h_{1} x+\bar{x} h_{1} y^{-1}=h_{1} x+\bar{y} h_{1} y^{-1}=h_{1} x-h_{1} y=0,
$$


whence $x y^{-1} \in d_{2} h_{2}^{-1}(0)$. This proves (b).

Let $c \in C_{1}$ be such that $\partial_{1} c=(\bar{x}-1) c^{0}$. Then $\partial_{1}\left(c-h_{1} x\right)=0$ and it follows from (a) that $c-h_{1} x=h_{1} y$ for some $y \in d_{2} \rho_{2}$. Let $x^{\prime}=y x$. Then $\bar{y}=1, x^{\prime} \in \bar{x}$ and

$$
h_{1} x^{\prime}=h_{1} y+\bar{y} h_{1} x=h_{1} y+h_{1} x=c,
$$

which completes the proof of Theorem 8 .

We now define $\partial_{2}$ by (8.1b), with $n=2$. Since $d_{2} h_{2}^{-1}(0)=1$ this gives a single-valued map, $\partial_{2}: C_{2} \rightarrow C_{1}$, which is obviously an operator homomorphism. Thus $\partial_{n}$ is defined for each $n \geqq 1$. It follows from (8.1b) and Theorem 8(a) that

$$
\partial_{1} \partial_{2} h_{2}=\partial_{1} h_{1} d_{2}=0
$$

and from (8.1b) that

$$
\partial_{n} \partial_{n+1} h_{n+1}=\partial_{n} h_{n} d_{n+1}=h_{n-1} d_{n} d_{n+1}=0 \quad(n \geqq 2) .
$$

Since $h_{n}$ is onto $C_{n}(n \geqq 2)$ it follows that $\partial \partial=0$.

Let $c \in C$ and $x \in \rho_{1}$ be given. Then we have:

Lemma 5. If $\partial_{2} c=h_{1} x$ there is a unique element, $a \in \rho_{2}$, such that $h_{2} a=c, d_{2} a=x$.

Since $h_{2}$ is onto $C_{2}$ there is an $a^{\prime} \in \rho_{2}$ such that $h_{2} a^{\prime}=c$. It follows from (8.1b) and the relation $\partial_{2} c=h_{1} x$ that

$$
h_{1} x=\partial_{2} c=\partial_{2} h_{2} a^{\prime}=h_{1} d_{2} a^{\prime} .
$$

Therefore it follows from Theorem $8(\mathrm{~b})$ that

$$
x=\left(d_{2} b\right)\left(d_{2} a^{\prime}\right)=d_{2}\left(b+a^{\prime}\right),
$$

where $b \in h_{2}^{-1}(0)$. Let $a=b+a^{\prime}$. Then $h_{2} a=h_{2} a^{\prime}=c$, since $h_{2} b=0$. Therefore $h_{2} a=c, d_{2} a=x$. Let $h_{2} a_{1}=c, d_{2} a_{1}=x$. Then $h_{2}\left(a-a_{1}\right)=0$, $d_{2}\left(a-a_{1}\right)=1$ and it follows from Lemma 1 that $a_{1}=a$. Therefore $a$ is unique.

9. Chain mappings and homomorphisms. Let $\rho, \rho^{\prime}$ be two homotopy systems and $C, C^{\prime}$ the associated systems of chain groups. By a chain mapping, $g: C \rightarrow C^{\prime}$, we shall mean a family of operator homomorphisms, $g_{n}: C_{n} \rightarrow C_{n}^{\prime}$, associated with a homomorphism, $\bar{f}: \bar{\rho}_{1} \rightarrow \bar{\rho}_{1}^{\prime}$, such that

$$
\begin{aligned}
\text { (a) } \quad g_{0} c^{0} & =c^{\prime}, \\
\text { (b) } g_{n-1} \partial_{n} & =\partial_{n} g_{n} \quad(n \geqq 1),
\end{aligned}
$$

where $c^{0}, c^{\prime 0}$ are the preferred basis elements in $C_{0}, C_{0}^{\prime}$. If there is a 
homomorphism, $f: \rho \rightarrow \rho^{\prime}$, such that

$$
g_{n} h_{n}=h_{n} f_{n}
$$

then we shall say that $f$ induces or realizes the chain mapping $g$. In this case it follows from (8.1a), (9.2) and the relation $g \bar{x}=(\bar{f} \bar{x}) g$ $\left(\bar{x} \in \bar{\rho}_{1}\right)$ that

$$
\begin{aligned}
\left(\bar{f}_{1} \bar{x}-1\right) c^{\prime 0} & =\partial_{1} h_{1} f_{1} x=\partial_{1} g_{1} h_{1} x \\
& =g_{0} \partial_{1} h_{1} x=g_{0}(\bar{x}-1) c^{0} \\
& =(\bar{f} \bar{x}-1) c^{\prime 0} .
\end{aligned}
$$

Therefore $\bar{f}=\bar{f}_{1}$.

Let $C, C^{\prime}, C^{\prime \prime}$ be systems of chain groups associated with homotopy systems $\rho, \rho^{\prime}, \rho^{\prime \prime}$. Let $g: C \rightarrow C^{\prime}, g^{\prime}: C^{\prime} \rightarrow C^{\prime \prime}$ be chain mappings which are induced by homomorphisms $f: \rho \rightarrow \rho^{\prime}, f^{\prime}: \rho^{\prime} \rightarrow \rho^{\prime \prime}$. Then it is easily verified that $g^{\prime} g: C \rightarrow C^{\prime \prime}$ is a chain mapping induced by $f^{\prime} f: \rho \rightarrow \rho^{\prime \prime}$.

Theorem 9. A homomorphism, $f: \rho \rightarrow \rho^{\prime}$, induces a unique chain mapping, $g: C \rightarrow C^{\prime}$. A chain mapping, $g: C \rightarrow C^{\prime}$, can be realized by at least one ${ }^{21}$ homomorphism, $f: \rho \rightarrow \rho^{\prime}$.

Let $f: \rho \rightarrow \rho^{\prime}$ be given. Then we define $g_{0}$ by $g_{0} \bar{x} c^{0}=\left(\bar{f}_{1} \bar{x}\right) c^{\prime 0}$. Let $\left\{x_{i}\right\}$ be a basis for $\rho_{1}$. Then $\left\{h_{1} x_{i}\right\}$ is a basis for $C_{1}$ and we define $g_{1}$ by $g_{1} h_{1} x_{i}=h_{1} f_{1} x_{i}$. It is easily verified that $g_{1} h_{1}, h_{1} f_{1}: \rho_{1} \rightarrow C_{1}^{\prime}$ are both crossed homomorphisms associated with $f_{1}$. Therefore $g_{1} h_{1}=h_{1} f_{1}$, according to Lemma 3.

If $n \geqq 2$ we define $g_{n}$ by $g_{n} h_{n}=h_{n} f_{n}$. If $h_{2} a=0$, then $a \in \rho_{2}^{c}$ and $f_{2} a \in \rho_{2}^{\prime c}$, whence $h_{2} f_{2} a=0$. Therefore $g_{2}$ is a single-valued map, which is obviously an operator homomorphism associated with $f_{1}$. So is $g_{n}=h_{n} f_{n} h_{n}^{-1}$ $(n>2)$. The family of maps $g_{n}: C_{n} \rightarrow C_{n}^{\prime}$ satisfies (9.2). Since $h_{n}$ is onto $C_{n}$ if $n>1$ and $\left\{h_{1} x_{i}\right\}$ is a basis for $C_{1}$ it follows that (9.2), together with $\bar{f}_{1}$ and (9.1a), determine $\left\{g_{n}\right\}$ uniquely.

It follows from (9.2), with $n=1$, and (8.1) that

$$
\begin{aligned}
\partial_{1} g_{1} h_{1} x & =\partial_{1} h_{1} f_{1} x=\left(\bar{f}_{1} \bar{x}-1\right) c_{0}^{\prime} \\
& =g_{0}(\bar{x}-1) c^{0} \\
& =g_{0} \partial_{1} h_{1} x .
\end{aligned}
$$

Therefore $\partial_{1} g_{1}=g_{0} \partial_{1}$. A similar, but simpler, argument shows that $\partial_{n} g_{n}=g_{n-1} \partial_{n}$ for any $n>1$. Therefore $g=\left\{g_{n}\right\}$ is a (uniquely determined) chain mapping, which is induced by $f$.

Now let $g: C \rightarrow C^{\prime}$ be a given chain mapping, associated with a

${ }^{21}$ See the addendum and (10.9) below. 
homomorphism $\bar{f}_{1}: \bar{\rho}_{1} \rightarrow \bar{\rho}_{1}^{\prime}$. Since

$$
\begin{aligned}
\partial_{1} g_{1} h_{1} x_{i} & =g_{0} \partial_{1} h_{1} x_{i}=g_{0}\left(\bar{x}_{i}-1\right) c^{0} \\
& =\left(\bar{f}_{1} \bar{x}_{i}-1\right) c^{\prime 0}
\end{aligned}
$$

it follows from Theorem 8 (c), applied to $\bar{\rho}_{1}^{\prime}$, that there is an element, $y_{i}^{\prime} \in \rho_{1}^{\prime}$, such that $g_{1} h_{1} x_{i}=h_{1} y_{i}^{\prime}$. We define $f_{1}: \rho_{1} \rightarrow \rho_{1}^{\prime}$ by $f_{1} x_{i}=y_{i}^{\prime}$. Then it follows from Lemma 3 , as in the first half of the proof, that

$$
g_{1} h_{1}=h_{1} f_{1} \text {. }
$$

Let $A$ be a basis for $\rho_{2}$ and let

$$
c_{a}^{\prime}=g_{2} h_{2} a \in C_{2}^{\prime}
$$

for each $a \in A$. Then

$$
\partial_{2} c_{a}^{\prime}=\partial_{2} g_{2} h_{2} a=g_{1} \partial_{2} h_{2} a=g_{1} h_{1} d_{2} a
$$

and it follows from (9.4) that

$$
\partial_{2} c_{a}^{\prime}=h_{1} f_{1} d_{2} a .
$$

By Lemma 5 there is a (unique) $b_{a}^{\prime} \in \rho_{2}^{\prime}$ such that $h_{2} b_{a}^{\prime}=c_{a}^{\prime}, d_{2} b_{a}^{\prime}$ $=f_{1} d_{2} a$. Since $d_{2} b_{a}^{\prime}=f_{1} d_{2} a$ it follows from Lemma 2 that there is a (unique) operator homomorphism, $f_{2}: \rho_{2} \rightarrow \rho_{2}^{\prime}$, associated with $f_{1}$, such that $f_{2} a=b_{a}^{\prime}$ for each $a \in A$. Then $d_{2} f_{2} a=f_{1} d_{2} a$ and

$$
h_{2} f_{2} a=h_{2} b_{a}^{\prime}=c_{a}^{\prime}=g_{2} h_{2} a .
$$

Therefore $d_{2} f_{2}=f_{1} d_{2}$ and $h_{2} f_{2}=g_{2} h_{2}$.

If $n \geqq 3$ let $f_{n}=h_{n}^{-1} g_{n} h_{n}$. Then $f_{n}(n \geqq 2)$ is an operator homomorphism associated with $f_{1}$ and $g h=h f, d_{2} f_{2}=f_{1} d_{2}$. It remains to prove that $d_{n} f_{n}=f_{n-1} d_{n}$ when $n>2$. It follows from the relations $h d=\partial h$, $h f=g h, \partial g=g \partial$ that

$$
\begin{aligned}
h_{n-1} d_{n} f_{n} & =\partial_{n} h_{n} f_{n}=\partial_{n} g_{n} h_{n} \\
& =g_{n-1} \partial_{n} h_{n}=g_{n-1} h_{n-1} d_{n} \\
& =h_{n-1} f_{n-1} d_{n} .
\end{aligned}
$$

If $n>3$, then $h_{n-1}$ is an isomorphism and it follows that $d_{n} f_{n}=f_{n-1} d_{n}$. Also $h_{2}\left(d_{3} f_{3}-f_{2} d_{3}\right)=0$ and

$$
d_{2}\left(d_{3} f_{3}-f_{2} d_{3}\right)=d_{2} d_{3} f_{3}-f_{1} d_{2} d_{3}=0 .
$$

Therefore it follows from Lemma 1 that $d_{3} f_{3}-f_{2} d_{3}=0$, which completes the proof.

There is an element of arbitrariness in the choice of $f_{1}$, satisfying (9.4). Let $f_{1}: \rho_{1} \rightarrow \rho_{1}^{\prime}$ be a given homomorphism such that $h_{1} f_{1}=g_{1} h_{1}$. 
Then the above construction gives $f: \rho \rightarrow \rho^{\prime}$ uniquely. Therefore we have the addendum to Theorem 9:

ADDENDUM. The chain mapping $g$ has a unique realization, $f$, such that $f_{1}: \rho_{1} \rightarrow \rho_{1}^{\prime}$ is a given homomorphism, which satisfies the condition $h_{1} f_{1}=g_{1} h_{1}$.

10. Chain homotopy. Let $\rho, \rho^{\prime}$ be given homotopy systems and $C, C^{\prime}$ the associated systems of chain groups. By a chain deformation operator: $\eta: C \rightarrow C^{\prime}$, associated with a homomorphism, $\bar{f}_{1}: \bar{\rho}_{1} \rightarrow \bar{\rho}_{1}$, we shall mean a family of operator homomorphisms, $\eta_{n}: C_{n-1} \rightarrow C_{n}$ $(n \geqq 1)$, associated with $\bar{f}_{1}$. Let $g, g^{\prime}: C \rightarrow C^{\prime}$ be chain mappings associated with homomorphisms $\bar{f}_{1}, \bar{f}_{1}^{\prime}: \bar{\rho}_{1} \rightarrow \bar{\rho}_{1}^{\prime}$. We shall describe $g$ as chain homotopic to $g^{\prime}$, and shall write $g \simeq g^{\prime}$, if, and only if, there is an element $v \bar{w}^{\prime} \in \bar{\rho}_{1}^{\prime}$ and a chain deformation operator, $\eta: C \rightarrow C^{\prime}$, associated with $\bar{f}_{1}$ such that

$$
\bar{w}^{\prime} g_{r}^{\prime}-g_{r}=\partial_{r+1} \eta_{r+1}+\eta_{r} \partial_{r}
$$$$
\left(r \geqq 0 ; \eta_{0} \partial_{0}=0\right) .
$$

Since $g_{0} c^{0}=g_{0}^{\prime} c^{\prime 0}=c^{\prime 0}$, where $c^{0} \in C_{0}, c^{\prime 0} \in C_{0}^{\prime}$ are the preferred basis elements, it follows from $(10.1)_{0}$ that $\partial_{1} \eta_{1} c^{0}=\left(\bar{w}^{\prime}-1\right) c^{\prime 0}$. It follows from Theorem 8(c) that there is a $w^{\prime} \in \bar{w}^{\prime}$ such that

$$
\eta_{1} c^{0}=h_{1} w^{\prime} \text {. }
$$

I say that, in consequence of $(10.1)_{0}$,

$$
\bar{f}_{1}^{\prime} \bar{x}=\bar{w}^{\prime-1}\left(\bar{f}_{1} \bar{x}\right) \bar{w}^{\prime} .
$$

For $\partial_{1} \eta_{1}: C_{0} \rightarrow C_{0}^{\prime}$ is an operator homomorphism associated with $\bar{f}_{1}$. Therefore it follows from $(10.1)_{0}$ that

$$
\begin{aligned}
\left(\bar{w}^{\prime} g_{0}^{\prime}-g_{0}\right) \bar{x} c^{0} & =\left(\bar{f}_{1} \bar{x}\right)\left(\bar{w}^{\prime} g_{0}^{\prime}-g_{0}\right) c^{0} \\
& =\left(\bar{f}_{1} \bar{x}\right)\left(\bar{w}^{\prime}-1\right) c^{\prime 0} .
\end{aligned}
$$

But we also have

$$
\begin{aligned}
\left(\bar{w}^{\prime} g_{0}^{\prime}-g_{0}\right) \bar{x} c^{0} & =\left\{\bar{w}^{\prime}\left(\bar{f}_{1}^{\prime} \bar{x}\right) g_{0}^{\prime}-\left(\bar{f}_{1} \bar{x}\right) g_{0}\right\} c^{0} \\
& =\left\{\bar{w}^{\prime}\left(\bar{f}_{1}^{\prime} \bar{x}\right)-\bar{f}_{1} \bar{x}\right\} c^{\prime 0} .
\end{aligned}
$$

Therefore

$$
\left(\bar{f}_{1} \bar{x}\right)\left(\bar{w}^{\prime}-1\right)=\bar{w}^{\prime}\left(\bar{f}_{1}^{\prime} \bar{x}\right)-\bar{f}_{1} \bar{x}
$$

which reduces to (10.3).

Let $g, g^{\prime}: C \rightarrow C^{\prime}$ be the chain mappings induced by homomorphisms $f, f^{\prime}: \rho \rightarrow \rho^{\prime}$. For convenience we display the formula

$$
w^{\prime} f_{n}^{\prime}-f_{n}=d_{n+1} \xi_{n+1}+\xi_{n} d_{n}
$$


in which $w^{\prime} \in \rho_{1}$, and $\xi: \rho \rightarrow \rho^{\prime}$ is a deformation operator.

THeOREM 10. $f \simeq f^{\prime}$ if, and only if, $g \simeq g^{\prime}$. More precisely, $f, f^{\prime}$ are related by (10.4) if, and only if, $g, g^{\prime}$ are related by (10.1), where

(a) $\eta_{1} c^{0}=h_{1} w^{\prime}$,

(b) $\quad h_{n} \xi_{n}=\eta_{n} h_{n-1}$

$(n \geqq 2)$.

First let $f \simeq f^{\prime}$ and let $f, f^{\prime}$ be related by (10.4). Let $\left\{x_{i}\right\}$ be a basis for $\rho_{1}$. Let $\eta_{1}: C_{0} \rightarrow C_{1}^{\prime}, \eta_{2}: C_{1} \rightarrow C_{2}^{\prime}$ be the operator homomorphisms, associated with $\bar{f}_{1}$, which are defined by $\eta_{1} c^{0}=h_{1} w, \eta_{2} h_{1} x_{i}=h_{2} \xi_{2} x_{i}$. Clearly $\eta_{2} h_{1}, h_{2} \xi_{2}: \rho_{1} \rightarrow C_{2}^{\prime}$ are both crossed homomorphisms associated with the homomorphism $x \rightarrow \bar{f}_{1} \bar{x}$. Therefore $\eta_{2} h_{1}=h_{2} \xi_{2}$. Since $\rho_{3}^{\prime}$ is Abelian it follows that $\xi_{3} h_{2}^{-1}(0)=0$. Therefore a single-valued map, $\eta_{3}: C_{2} \rightarrow C_{3}^{\prime}$, which is obviously an operator homomorphism, is defined by $\eta_{3} h_{2}=h_{3} \xi_{3}$. Let $\eta_{n}=h_{n} \xi_{n} h_{n-1}^{-1}$ if $n>3$. Then (10.5) is satisfied.

Let $F_{n}: \rho_{n} \rightarrow \rho_{n}^{\prime}, G_{r}: C_{r} \rightarrow C_{r}^{\prime}$ be the maps which are given by

$$
\begin{aligned}
& F_{n}=w^{\prime} f_{n}{ }^{\prime}-f_{n}-\xi_{n} d_{n}-d_{n+1} \xi_{n+1} \\
& (n \geqq 1) \text {, } \\
& G_{r}=\bar{w}^{\prime} g_{r}^{\prime}-g_{r}-\eta_{r} \partial_{r}-\partial_{r+1} \eta_{r+1} \\
& (r \geqq 0) \text {. }
\end{aligned}
$$

We shall prove that

$$
h_{n} F_{n}=G_{n} h_{n},
$$

assuming only (10.3), (10.5) and that $g, g^{\prime}$ are induced by $f, f^{\prime}$. Since $h f=g h$ we have

$$
h_{n}\left(w^{\prime} f_{n}^{\prime}-f_{n}\right)=\left(\bar{w}^{\prime} g_{n}^{\prime}-g_{n}\right) h_{n} \quad(n \geqq 2)
$$

and from (8.1) and (10.5)

$$
\begin{aligned}
h_{n}\left(d_{n+1} \xi_{n+1}+\xi_{n} d_{n}\right) & =\partial_{n+1} h_{n+1} \xi_{n+1}+\eta_{n} h_{n-1} d_{n} \\
& =\left(\partial_{n+1} \eta_{n+1}+\eta_{n} \partial_{n}\right) h_{n} .
\end{aligned}
$$

Therefore $(10.7)$ is valid if $n \geqq 2$.

Let $x \in \rho_{1}$ and let $u=f_{1} x, u^{\prime}=f_{1}^{\prime} x$. Then $\overline{w^{\prime} u^{\prime} w^{\prime}}-1=u$ according to (10.3), and it follows from (3.2) and (3.3) that

$$
\begin{aligned}
h_{1}\left(w^{\prime} u^{\prime} w^{-1} w^{-1}\right) & =h_{1} w^{\prime}+w^{\prime} h_{1} u^{\prime}-\bar{u} h_{1} w^{\prime}-h_{1} u \\
& =\bar{w}^{\prime} h_{1} u^{\prime}-h_{1} u-(\bar{u}-1) h_{1} w^{\prime} \\
& =\bar{w}^{\prime} h_{1} f_{1}^{\prime} x-h_{1} f_{1} x-\left(\bar{f}_{1} \bar{x}-1\right) \eta_{1} c^{0} \\
& =\left(\bar{w}^{\prime} g_{1}^{\prime}-g_{1}\right) h_{1} x-\eta_{1}(\bar{x}-1) c^{0} \\
& =\left(\bar{w}^{\prime} g_{1}^{\prime}-g_{1}\right) h_{1} x-\eta_{1} \partial_{1} h_{1} x \\
& =G_{1} h_{1} x+\partial_{2} \eta_{2} h_{1} x .
\end{aligned}
$$


But $\partial_{2} \eta_{2} h_{1}=\partial_{2} h_{2} \xi_{2}=h_{1} d_{2} \xi_{2}$. Since

$$
\left(w^{\prime} u^{\prime} w^{\prime-1} u^{-1}\right)^{*}=1, \quad\left(d_{2} \xi_{2} x\right)^{*}=1,
$$

where $y^{*}=\bar{y}\left(y \in \rho_{1}^{\prime}\right)$, it follows that

$$
\begin{aligned}
h_{1} F_{1} x & =h_{1}\left\{w^{\prime} u^{\prime} w^{-1} u^{-1}\left(d_{2} \xi_{2} x\right)^{-1}\right. \\
& =h_{1}\left(w^{\prime} u^{\prime} w^{-1} u^{-1}\right)-h_{1} d_{2} \xi_{2} x \\
& =G_{1} h_{1} x .
\end{aligned}
$$

Therefore (10.7) is valid for every $n \geqq 1$. Since $h_{n} \rho_{n}=C_{n}$ if $n>1$ and $\left\{h_{1} x_{i}\right\}$ is a basis for $C_{1}$ it follows that $G_{n}=0$ in consequence of $F_{n}=0$. Since $g_{0} c^{0}=g_{0}^{\prime} c^{0}=c^{\prime 0}$ it follows from $(10.5 \mathrm{a})$ that $G_{0} c^{0}=\left(\bar{w}^{\prime}-1\right) c^{\prime 0}$ $-\partial_{1} \eta_{1} c^{0}=0$. Therefore $(10.4)$ implies $(10.1)$, with $\xi$ and $\eta$ related by (10.5).

Conversely let $g, g^{\prime}$ be related by (10.1). Then (10.2), i.e. (10.5a), and (10.3) are satisfied. Let $u_{i}=f_{1} x_{i}, u_{i}^{\prime}=f_{1}^{\prime} x_{i}$. Then it follows from (10.8), with $G_{1}=0$, and Lemma 5 that there is a (unique) element, $a_{i}^{\prime} \in \rho_{2}^{\prime}$, such that

$$
h_{2} a_{i}^{\prime}=\eta_{2} h_{1} x_{i}, \quad d_{2} a_{i}^{\prime}=w^{\prime} u^{\prime} w^{\prime-1} u^{-1} .
$$

Let $\xi_{2}: \rho_{1} \rightarrow \rho_{2}^{\prime}$ be the crossed homomorphism which is determined by $\xi_{2} x_{i}=a_{i}^{\prime}$, according to Lemma 3 . Then, reverting to the additive notation for $\rho_{1}^{\prime}$, we have

$$
h_{2} \xi_{2} x_{i}=\eta_{2} h_{1} x_{i}, \quad d_{2} \xi_{2} x_{i}=w^{\prime} f_{1}^{\prime} x_{i}-f_{1} x_{i} .
$$

Clearly $h_{2} \xi_{2}, \eta_{2} h_{1}: \rho_{1} \rightarrow C_{2}^{\prime}$ are crossed homomorphisms associated with the homomorphism $x \rightarrow \bar{f}_{1} \bar{x}$. Also $d_{2} \xi_{2}: \rho_{1} \rightarrow \rho_{1}^{\prime}$ and, as observed in $\S 3$, $\left(w^{\prime} f_{1}^{\prime}-f_{1}\right): \rho_{1} \rightarrow \rho_{1}^{\prime}$ are crossed homomorphisms associated with $f_{1}$. Therefore

$$
h_{2} \xi_{2}=\eta_{2} h_{1}, \quad d_{2} \xi_{2}=w^{\prime} f_{1}^{\prime}-f_{1}
$$

If $n>2$ we define

$$
\xi_{n}=h_{n}^{-1} \eta_{n} h_{n-1}: \rho_{n-1} \rightarrow \rho_{n}^{\prime},
$$

thus defining a deformation operator $\xi$, which satisfies (10.5b). Since (10.3) and (10.5) are satisfied, so is (10.7). Since $G_{n}=0$ it follows that $h_{n} F_{n}=0$, whence $F_{n}=0$ if $n>2$.

Since

$$
d_{n}\left(w^{\prime} f_{n}^{\prime}-f_{n}\right)=\left(w^{\prime} f_{n-1}^{\prime}-f_{n-1}\right) d_{n}
$$

and

$$
d_{n}\left(d_{n+1} \xi_{n+1}+\xi_{n} d_{n}\right)=d_{n} \xi_{n} d_{n}=\left(d_{n} \xi_{n}+\xi_{n-1} d_{n-1}\right) d_{n}
$$


it follows that

$$
d_{n} F_{n}=F_{n-1} d_{n} \quad(n \geqq 2) .
$$

We have proved that $F_{1}=0$, whence $d_{2} F_{2}=0$. Since $h_{2} F_{2}=0$ it follows from Lemma 1 that $F_{2}=0$. Therefore $F_{n}=0$ for $n \geqq 2$ and the theorem is proved.

On taking $g=g^{\prime}, \eta=0, w^{\prime}=1$ we have the corollary:

Corollary. If $f, f^{\prime}: \rho \rightarrow \rho^{\prime}$ induce the same chain mapping, $g: C \rightarrow C^{\prime}$, then $f \simeq f^{\prime}$.

In this case it follows from (10.5) that $\xi_{n}=0$ if $n>2$. Also we may take $w^{\prime}=1$, so that

$$
f_{1}^{\prime}-f_{1}=d_{2} \xi_{2}, \quad f_{2}^{\prime}-f_{2}=\xi_{2} d_{2}, \quad f_{n}^{\prime}=f_{n} \quad(n>2),
$$

where $h_{2} \xi_{2}=\eta_{2} h_{1}=0$.

The operator $\eta$ is determined uniquely by (10.5) if $w^{\prime}$ and $\xi$ are given. When $\eta$ is given the element $w^{\prime}$ may be replaced by $z^{\prime} w^{\prime}$, for any $z^{\prime} \in d_{2} h_{2}^{-1}(0) \subset \rho_{1}^{\prime}$, according to Theorem $8(\mathrm{~b})$. Once $w^{\prime}$ has been fixed, so as to satisfy (10.2), then $\xi$ is determined uniquely by the conditions $F_{1}=0$ and (10.5). Moreover in this statement it need only be assumed that $\xi_{n}: \rho_{n-1} \rightarrow \rho_{n}^{\prime}$ is a single-valued transformation. For $\xi_{2} x \in \rho_{2}^{\prime}$ is determined uniquely by the conditions

$$
d_{2} \xi_{2} x=\left(w^{\prime} f_{1}^{\prime}-f_{1}\right) x, \quad h_{2} \xi_{2} x=\eta_{2} h_{1} x
$$

and $\xi_{n}$ by $\xi_{n}=h_{n}^{-1} \eta_{n} h_{n-1}(n \geqq 3)$. But we have proved that there is a deformation operator, $\xi: \rho \rightarrow \rho^{\prime}$, associated with $f_{1}$, which satisfies these conditions. Therefore the fact that $\xi_{2}$ is a crossed homomorphism associated with $f_{1}$, and $\xi_{n}$ an operator homomorphism if $n>2$, is a consequence of $F_{1}=0$ and (10.5).

11. Chain equivalence: Proof of Theorem 1. Let $C, C^{\prime}$ be systems of chain groups associated with homotopy systems $\rho, \rho^{\prime}$. A chain mapping, $g: C \rightarrow C^{\prime}$, is called a chain equivalence if, and only if, there is a chain mapping, $g^{\prime}: C^{\prime} \rightarrow C$, such that $g^{\prime} g \simeq 1, g g^{\prime} \simeq 1$. We shall describe $C$ as equivalent to $C^{\prime}$, and shall write $C \equiv C^{\prime}$, if, and only if, there is a chain equivalence $g: C \rightarrow C^{\prime}$.

THEOREM 11 . The relations $\simeq$, between chain mappings, and $\equiv$, between systems of chain groups, are equivalence relations.

On taking $\bar{w}^{\prime}=1, \eta=0$ in (10.1) we have $g \simeq g$. In general it follows from (10.1) that

$$
\bar{w}^{\prime-1} g-g^{\prime}=\partial \eta^{*}+\eta^{*} \partial
$$


where $\eta^{*}=-\bar{w}^{\prime-1} \eta$. Since $\eta \bar{x}=\left(\bar{f}_{1} \bar{x}\right) \eta$ it follows from (10.3) that

$$
\eta^{*} \bar{x}=-\bar{w}^{\prime-1}\left(\bar{f}_{1} \bar{x}\right) \eta=-\left(\bar{f}_{1}^{\prime} \bar{x}\right) \bar{w}^{\prime-1} \eta^{*} .
$$

Therefore $\eta^{*}$ is a chain deformation operator associated with $\bar{f}_{1}^{\prime}$ and it follows that $g^{\prime} \simeq g$.

Let $g, g^{\prime}: C \rightarrow C^{\prime}$ be related by (10.1) and let $g^{\prime}, g^{\prime \prime}: C \rightarrow C^{\prime}$ be related by

$$
\bar{w}^{\prime \prime} g^{\prime \prime}-g^{\prime}=\partial \eta^{\prime}+\eta^{\prime} \partial,
$$

where $\eta^{\prime}$ is a chain deformation operator associated with $\bar{f}_{1}^{\prime}$. Then

$$
\bar{w}^{\prime} \bar{w}^{\prime \prime} g^{\prime \prime}-g=\partial\left(\eta+\bar{w}^{\prime} \eta^{\prime}\right)+\left(\eta+\bar{w}^{\prime} \eta^{\prime}\right) \partial .
$$

Moreover

$$
\begin{aligned}
\left(\eta+\bar{w}^{\prime} \eta^{\prime}\right) \bar{x} & =\left(\bar{f}_{1} \bar{x}\right) \eta+\bar{w}^{\prime}\left(\bar{f}_{1}^{\prime} \bar{x}\right) \eta^{\prime} \\
& =\left(\bar{f}_{1} \bar{x}\right)\left(\eta+\bar{w}^{\prime} \eta^{\prime}\right) .
\end{aligned}
$$

Therefore $\eta+\bar{w}^{\prime} \eta^{\prime}$ is a chain deformation operator associated with $\bar{f}_{1}$. Therefore $g \simeq g^{\prime}$ and $g^{\prime} \simeq g^{\prime \prime}$ together imply $g \simeq g^{\prime \prime}$. Therefore $\simeq$ is an equivalence relation.

The relation $\equiv$ is obviously reflexive and symmetric. Let $C, C^{\prime}, C^{\prime \prime}$ be chain groups associated with homotopy systems $\rho, \rho^{\prime}, \rho^{\prime \prime}$. Let $f \simeq g: C \rightarrow C^{\prime}, f^{\prime} \simeq g^{\prime}: C^{\prime} \rightarrow C^{\prime \prime}$. Then it is easily verified that

$$
f^{\prime} f \simeq f^{\prime} g \simeq g^{\prime} g
$$

It follows from this, as in the ordinary theory of homotopy or chain homotopy, that $\equiv$ is transitive. This completes the proof.

Let $\rho, \rho^{\prime}$ be given homotopy systems and $C, C^{\prime}$ associated systems of chain groups.

Theorem 12. $\rho \equiv \rho^{\prime}$ if, and only if, $C \equiv C^{\prime}$. More precisely, a homomorphism, $f: \rho \rightarrow \rho^{\prime}$, is an equivalence if, and only if, the induced chain mapping, $g: C \rightarrow C^{\prime}$, is a chain equivalence.

Let $f: \rho \rightarrow \rho^{\prime}$ and $f^{\prime}: \rho^{\prime} \rightarrow \rho$ be homomorphisms and let $g: C \rightarrow C^{\prime}$ and $g^{\prime}: C^{\prime} \rightarrow C$ be the induced chain mappings. Then $g^{\prime} g, g g^{\prime}$ are induced by $f^{\prime} f, f f^{\prime}$. Also the identical chain mappings, $1: C, C^{\prime} \rightarrow C, C^{\prime}$ are obviously induced by $1: \rho, \rho^{\prime} \rightarrow \rho, \rho^{\prime}$. Therefore it follows from Theorem 10 that $f^{\prime} f \simeq 1, f f^{\prime} \simeq 1$ if, and only if, $g^{\prime} g \simeq 1, g g^{\prime} \simeq 1$. This proves the theorem.

As an obvious corollary of Theorems 11 and 12 we have one half of Theorem 1 , namely that $\equiv$ is an equivalence relation between homotopy systems. To prove the other half let $f \simeq f^{\prime}: \rho \rightarrow \rho^{\prime}$ and let $g, g^{\prime}: C \rightarrow C^{\prime}$ be the induced chain mappings. Then it follows from 
Theorems 10 and 11 that $g \simeq g^{\prime}, g^{\prime} \simeq g, f^{\prime} \simeq f$. Therefore the relation $\simeq$ is symmetric. Similarly it is transitive and it is obviously reflexive. Therefore it is an equivalence relation. This proves Theorem 1.

It follows from (10.3), and an argument similar to the one at the end of $\S 4$, that the homomorphism, $\vec{f}_{1}: \bar{\rho}_{1} \rightarrow \bar{\rho}_{1}^{\prime}$, which is associated with a chain equivalence, is necessarily an isomorphism.

12. Covering complexes. Let $\widetilde{K}$ be a universal covering complex of a given complex, $K$, with base point $e^{0} \in K^{0}$. Let $p: \widetilde{K} \rightarrow K$ be the covering map and let a 0 -cell $\tilde{e}^{0} \in p^{-1} e^{0}$ be taken as base point in $\tilde{K}$. We shall show how a system of chain groups $\left\{C_{n}\right\}$, associated with $\rho(K)$, may be "realized" as a system of chain groups in $\tilde{K}$.

Our conventions concerning homology are as follows. Let $X$ and $X_{0} \subset X$ be given spaces. Then $H_{n}\left(X, X_{0}\right)$ will denote the $n$th relative homology group, with integral coefficients, which is defined in terms of (finite) singular chains. By an oriented $n$-element, $E^{n}$, we shall mean an $n$-element, $E^{n}$, associated with a generator, $c$, of $H_{n}\left(E^{n}, \partial E^{n}\right)$. Let $E^{n}$ be thus oriented. Then a map

$$
\theta:\left(E^{n}, \partial E^{n}\right) \rightarrow\left(X, X_{0}\right)
$$

induces a homomorphism

$$
\theta_{*}: H_{n}\left(E^{n}, \partial E^{n}\right) \rightarrow H_{n}\left(X, X_{0}\right)
$$

and we shall described $\theta_{*} c$ as the element represented by $\theta$. Similar observations apply to $\partial E^{n}$ and maps $\partial E^{n} \rightarrow X_{0}$.

We orient $I$ so that 0 is its first and 1 is its last point. We orient $I^{n}=I \times I^{n-1}(n \geqq 1)$ and its faces by induction on $n$ and the standard rule for orienting topological products. This gives

$$
\partial I^{n+1}=1 \times I^{n}-0 \times I^{n}-I \times \partial I^{n} .
$$

Following S. Eilenberg and N. E. Steenrod we take

$$
C_{n}=C_{n}(K)=H_{n}\left(\widetilde{K}^{n}, \widetilde{K}^{n-1}\right)
$$

as the group of $n$-chains ${ }^{22}$ in $\tilde{K}$, with $C_{0}(K)=H_{0}\left(\tilde{K}^{0}\right)$. We shall use

$$
\tau(\bar{x}): \tilde{K} \rightarrow \tilde{K}
$$

to denote the covering transformation (Deckbewegung), which is determined by $\bar{x} \in \bar{\rho}_{1}=\pi_{1}(K)$. We shall also use $\bar{x}$ to denote the automorphism

${ }^{22}$ See [11] and also [13, §17]. All our chains are finite because of (D) and (N) in in $\S 5$ of $(\mathrm{I})$, from which it follows that any compact sub-set is contained in a finite sub-complex of $\widetilde{K}$. 


$$
\bar{x}: C_{n} \rightarrow C_{n},
$$

which is induced by $\tau(\bar{x})$. It satisfies the condition $\partial \bar{x}=\bar{x} \partial$. The group $C_{n}$, with $\bar{x}: C_{n} \rightarrow C_{n}$ thus defined, is a free $\bar{\rho}_{1}$-module.

Let $\left\{e_{i}^{n}\right\}$ be the cells in $K$ and let $\widetilde{e}_{i}^{n} \in \tilde{K}$ be a cell which covers $e_{i}^{n}$. The cell $\tilde{e}_{i}^{n}$ may be any one of those which cover $e_{i}^{n}$ unless $n=0$ and $e_{i}^{0}$ is the base point $e^{0} \in K^{0}$. In this case $\tilde{e}_{i}^{0}$ shall be the base point $\tilde{e}^{0} \in \tilde{K}^{0}$. Let $c_{i}^{n} \in C_{n}$ be an elementary $n$-chain associated with $\tilde{e}_{i}^{n}$, meaning the element represented by a characteristic map for $\widetilde{e}_{i}^{n}$. Then $\left\{c_{i}^{n}\right\}$ is a basis, which we call a natural basis, for $C_{n}$.

We proceed to show that the groups $C_{n}(n>1)$ are related to the groups $\rho_{n}=\rho_{n}(K)$ in the way described in $\S 8$. If $K^{0}$ consists of a single 0 -cell the same is true of $C_{0}, C_{1}$. In general we shall define sub-groups of $C_{0}, C_{1}$ which are thus related to $\rho_{0}, \rho_{1}$. We begin with some preliminary observations on lifted maps and homotopies.

Let $\phi:\left(K, e^{0}\right) \rightarrow\left(L, e^{\prime 0}\right)$ be a map into a complex, $L$, with base point $e^{\prime 0}$. Then there is a unique "lifted" map,

$$
\tilde{\phi}:\left(\tilde{K}, \tilde{e}^{0}\right) \rightarrow\left(\tilde{L}, \tilde{e}^{\prime 0}\right),
$$

such that $\phi p=p \tilde{\phi}$, where $\tilde{L}$ is a universal covering complex of $L$ and $\tilde{e}^{\prime 0} \in \widetilde{L}^{0}$ is the base point in $\tilde{L}$. Similarly a homotopy $\phi_{t}: K \rightarrow L$, such that $\phi_{0} e^{0}=e^{\prime 0}$, can be lifted into a unique homotopy, $\widetilde{\phi}_{t}: \widetilde{K} \rightarrow \tilde{L}$, such that $\phi_{t} \boldsymbol{p}=\boldsymbol{p} \widetilde{\boldsymbol{\phi}}_{t}, \tilde{\boldsymbol{\phi}}_{0} \tilde{e}^{0}=\tilde{e}^{\prime}$. When we refer to a lifted map, $\tilde{\boldsymbol{\phi}}$ or homotopy, $\widetilde{\phi}_{t}$, it is always to be understood that $\widetilde{\phi} \widetilde{e}^{0}=\widetilde{e}^{\prime 0}$ or $\widetilde{\phi}_{0} \tilde{e}^{0}=\tilde{e}^{\prime 0}$. Let $\phi_{t}$ be lifted into $\widetilde{\phi}_{t}$, and let $\phi_{1} e^{0}=e^{\prime 0}$, in addition to $\phi_{0} e^{0}=e^{\prime 0}$. Let $w \in \pi_{1}\left(L^{1}\right)$ be the element represented by $\theta: I \rightarrow L$, where $\theta(t)=\phi_{t} e^{0}$. Then $\widetilde{\phi}_{1} \tilde{e}^{0}=\tau(\bar{w}) \tilde{e}^{\prime 0}$, whence

$$
\tilde{\phi}_{1}=\tau(\bar{w}) \tilde{\phi}_{1}^{\prime},
$$

where $\tilde{\phi}_{1}^{\prime}$ is the map defined by lifting $\phi_{1}$. It is inherent in the lifting process $^{23}$ that

$$
\tilde{\phi}_{t} \tau(\bar{x})=\tau\left(\bar{f}_{1} \bar{x}\right) \tilde{\phi}_{t},
$$

where $\bar{x} \in \bar{\rho}_{1}$ and $\bar{f}_{1}: \pi_{1}(K) \rightarrow \pi_{1}(L)$ is the homomorphism induced by $\phi_{0}$.

Let $\lambda:\left(I^{n}, I^{0}\right) \rightarrow\left(K^{n}, e^{0}\right)(n \geqq 1)$ be a map which represents a given element $a \in \rho_{n}$. We define $h_{n} a \in C_{n}$ as the element represented by the lifted map $\tilde{\lambda}: I^{n} \rightarrow \widetilde{K}^{n}$. If $n>1$, then $h_{n}: \rho_{n} \rightarrow C_{n}$ is the resultant of the "lifting" isomorphism, $\rho_{n} \rightarrow \tilde{\rho}_{n}=\rho_{n}(\tilde{K})$, followed by the homomorphism $\widetilde{h}_{n}: \widetilde{\rho}_{n} \rightarrow C_{n}$, in which corresponding elements are represented by the same map. The latter is onto and its kernel is the commutator sub-

${ }^{23}$ This is obvious if the lifting is defined in terms of paths joining $e^{0}$ to arbitrary points in $K$. 
group of $\tilde{\rho}_{n}$, which is zero if $n>2$. Therefore $h_{n}$ is onto and its kernel is the commutator sub-group of $\rho_{n}$. As in $\$ 8$ above it follows that $h_{1}: \rho_{1} \rightarrow C_{1}$ is a crossed homomorphism and that

$$
\partial_{1} h_{1} x=(\bar{x}-1) c^{0},
$$

where $c^{0} \in C_{0}$ is the elementary 0 -chain associated with $\tilde{e}^{0}$.

We prove that $h_{n}(n \geqq 2)$ is an operator homomorphism. Let

$$
\theta:(I, 0,1) \rightarrow\left(K^{1}, e^{0}, e^{0}\right)
$$

be a map, which represents a given element $x \in \rho$, and let $\tilde{\theta}$ be the lifted map. Then $\tilde{\theta}(1)=\tau(\bar{x}) \tilde{e}^{0}$. If $a \in \rho_{n}$ is represented by a map, $\lambda_{1}: I^{n} \rightarrow K^{n}$, then $x a$ is represented by a map, $\lambda_{0}: I^{n} \rightarrow K^{n}$, which is related to $\lambda_{1}$ by a homotopy,

$$
\lambda_{t}:\left(I^{n}, \partial I^{n}\right) \rightarrow\left(K^{n}, K^{n-1}\right),
$$

such that $\lambda_{t} I^{0}=\theta(t)$. Let $\tilde{\lambda}_{t}$ be the lifted homotopy $\left(\tilde{\lambda}_{0} I^{0}=\tilde{e}^{0}\right)$ and let $\tilde{\lambda}^{\prime}$ be the map defined by lifting $\lambda_{1}$. Then $\tilde{\lambda}^{\prime}$ represents $h_{n} a$ and, as in (12.3), $\tilde{\lambda}_{1}=\tau(\bar{x}) \tilde{\lambda}^{\prime}$. Therefore $\tilde{\lambda}_{1}$ represents $\bar{x} h_{n} a$. But $h_{n}(x a)$ is represented by $\tilde{\lambda}_{0}$ and hence also by $\tilde{\lambda}_{1}$, since an element of $C_{n}$ is defined without reference to base points. Therefore

$$
h_{n}(x a)=\bar{x} h_{n} a \quad(n \geqq 2) .
$$

We now prove that $\partial h=h d$. Let

$$
\lambda:\left(I^{n}, \partial I^{n}, E^{n-1}\right) \rightarrow\left(K^{n}, K^{n-1}, e^{0}\right)
$$

be a map which defines $a \in \rho_{n}$, where

$$
E^{n-1}=\partial I^{n}-\left(I_{1}^{n-1}-\partial I_{1}^{n-1}\right) \quad\left(I_{1}^{n-1}=1 \times I^{n-1}\right) .
$$

Then $\beta_{n} a$ and $d_{n} a$ are represented by

$$
\mu k: I^{n-1} \rightarrow K^{n-1},
$$

where $\mu=\lambda \mid I_{1}^{n-1}$ and $k p=(1, p)$, as in $\S 5$. Let $\tilde{\lambda}$ be the map defined by lifting $\lambda$. Since $E^{n-1}$ is connected it follows that $\tilde{\lambda} E^{n-1}=\tilde{e}^{0}$, whence $\tilde{\lambda}\left(\partial I_{1}^{n-1}\right)=\tilde{e}^{0}$. Therefore $\mu$ lifts into $\tilde{\mu}=\tilde{\lambda} \mid I_{1}^{n-1}$ and $\mu k$ lifts into $\tilde{\mu} k$. Therefore $\tilde{\mu} k$ represents $h_{n-1} d_{n} a$. The element $\partial_{n} h_{n} a$ is represented by $\tilde{\lambda} \mid \partial I^{n}$ or, since $\tilde{\lambda} E^{n-1}=\tilde{e}^{0}$ and $n-1>0$, by $\tilde{\mu}$ or by $\tilde{\mu} k$. Therefore

$$
\partial_{n} h_{n}=h_{n-1} d_{n}
$$

Let $T \subset K^{1}$ be a tree, which is a sub-complex containing $K^{0}$, and let $\tilde{T} \subset \tilde{K}^{1}$ be the component of $p^{-1} T$ which contains the base point $\tilde{e}^{0}$. Let $C_{r}(\widetilde{T}) \subset C_{r}(r=0,1)$ be the group of $r$-chains carried by $\widetilde{T}$. 
Since $\pi_{1}(T)=1$ it follows that $p \mid \widetilde{T}$ is a homeomorphism onto $T$. Therefore $C_{r}(\widetilde{T})$ is an ordinary free Abelian group. Since $K^{0} \subset T$, a basis for $C_{0}(\tilde{T})$ is a basis for $C_{0}$. Let $C_{1}^{\prime} \subset C_{1}$ be the (free) $\bar{\rho}_{1}$-module, which is generated ${ }^{24}$ by a basis for $C_{1}(\widetilde{T})$. If $K^{1} \neq T$ let $\left\{e_{i}^{1}\right\}$ be the $1-$ cells in $K^{1}-T$ and let $g_{i}: I \rightarrow \bar{e}_{i}^{-1}$ be a characteristic map for $e_{i}^{1}$. Let $\theta_{i}: I \rightarrow K^{1}$ be the map which consists of a path in $T$, leading from $e^{0}$ to $g_{i}(0)$, followed by $g_{i}$, followed by a path in $T$, which leads from $g_{i}(1)$ back to $e^{0}$. Let $x_{i} \in \rho_{1}$ be the element defined by $\theta_{i}$. Then $\left\{x_{i}\right\}$ is a basis for $\rho_{1}$. Since $h_{1} x_{i} \in C_{1}$ is represented by the lifted map, $\tilde{\theta}_{i}$, it follows that

$$
h_{1} x_{i}=c_{\imath}+c_{i}^{\prime}
$$

where $c_{i}$ is defined by the map $\tilde{g}_{i}: I \rightarrow \widetilde{K}^{1}$, such that $g_{i}=p \tilde{g}_{i}, \tilde{g}_{i}(0) \in \widetilde{T}$, and $c_{i}^{\prime} \in C_{i}^{\prime}$ : The set $\left\{c_{i}\right\}$, together with a natural basis, $\left\{c_{\lambda}^{\prime}\right\}$, for $C_{1}^{\prime}$ constitutes a natural basis, $\left\{c_{i}, c_{\lambda}^{\prime}\right\}$, for $C_{1}$. Therefore $\left\{h_{1} x_{i}, c_{\lambda}^{\prime}\right\}$ is a basis for $C_{1}$. Hence $C_{1}$ is the direct sum

$$
C_{1}=C_{1}^{*}+C_{1}^{\prime}
$$

where $C_{1}^{*}$ is the (free) $\bar{\rho}_{1}$-module generated by $\left\{h_{1} x_{i}\right\}$. Let $C_{0}^{*} C C_{0}$ be the $\bar{\rho}_{1}$-module generated by the elementary 0 -chain, $c^{0}$, associated with $\tilde{e}^{0}$. Let $C_{n}^{*}=C_{n}$ if $n>1$. Then $h_{n} \rho_{n} \subset C_{n}^{*}$ and we interpret $h_{n}$ as a map $h_{n}: \rho_{n} \rightarrow C_{n}^{*}(n \geqq 1)$. It follows from (12.5), (12.6) and (12.7) that $\rho(K)$ is related by $h_{n}$ to the system $C^{*}=\left\{C_{n}^{*}\right\}$ in the way described in $\S 8$. We shall describe $C$ as the complete system and $C^{*}$ as a normalized system of chain groups determined by $K$. In general $C^{*}$ depends on the choice of $T$. If $K^{0}=T=e^{0}$ then $C^{*}=C$.

Lemma 6. $C_{1}^{*}=\partial^{-1} C_{0}^{*}$.

It follows from (12.5) that $\partial_{1} C_{1}^{*} \subset C_{0}^{*}$. We have to prove that, if $\partial_{1} c \in C_{0}^{*}\left(c \in C_{1}\right)$ then $c \in C_{1}^{*}$. Let $\left\{c_{\lambda}^{0}\right\}$ be the set of elementary 0 -chains associated with the 0 -cells in $\tilde{T}-\tilde{e}^{0}$. Let $v_{\lambda}=c_{\lambda}^{0}-c^{0}$. Then $\left\{c^{0}, v_{\lambda}\right\}$ is a basis for $C_{0}$. Therefore $C_{0}$ is the direct sum

$$
C_{0}=C_{0}^{*}+C_{0}^{\prime},
$$

where $C_{0}^{\prime}$ has $\left\{v_{\lambda}\right\}$ for a basis. Since $\tilde{T}$ is a tree there is a basis, $\left\{c_{\lambda}^{\prime}\right\}$, for $C_{1}(\widetilde{T})$ such that $\partial_{1} c_{\lambda}^{\prime}=v_{\lambda}$. Then $\left\{c_{\lambda}^{\prime}\right\}$ is a basis for the summand $C_{1}^{\prime}$ in (12.8) and $\partial_{1} \mid C_{1}^{\prime}$ is an isomorphism onto $C_{0}^{\prime}$ : Let $c=c^{*}$ $+c^{\prime}$, where $c^{*} \in C_{1}^{*}, c^{\prime} \in C_{1}^{\prime}$, and let $\partial_{1} c \in C_{0}^{*}$. Since $\partial_{1} C_{1}^{*} \subset C_{0}^{*}$, $\partial_{1} C_{1}^{\prime} \subset C_{0}^{\prime}$ it follows that $\partial_{1} c^{\prime}=0$. Therefore $c^{\prime}=0$ and $c \in C_{1}^{*}$.

\footnotetext{
${ }^{24}$ I.e. generated by $\bar{\rho}_{1}$, operating on the basis elements in $C_{1}(\widetilde{T})$.
} 
13. Chain mappings: Proof of Theorems 5 and 6. Let $\phi:\left(K, e^{0}\right)$ $\rightarrow\left(L, e^{\prime 0}\right)$ be a given map of $K$ in a complex $L$, with base point $e^{\prime 0}$. Let

$$
g_{n}: C(K) \rightarrow C(L) \quad(n=0,1, \cdots)
$$

be the homomorphism induced by the lifted map, $\tilde{\phi}: \tilde{K} \rightarrow \tilde{L}$. It follows from (12.4), with $\widetilde{\phi}_{t}=\tilde{\phi}$ that each $g_{n}$ is an operator homomorphism associated with the homomorphism, $\bar{f}_{1}: \pi_{1}(K) \rightarrow \pi_{1}(L)$, which is induced by $\phi$. Also $g \partial=\partial g$ and we shall describe

$$
g=\left\{g_{n}\right\}: C(K) \rightarrow C(L)
$$

as the chain mapping induced by $\tilde{\phi}$. We shall also describe it as $i n$ duced, or realized geometrically by $\phi$. A chain mapping, $g: C(K) \rightarrow C(L)$, which need not have a geometrical realization, shall be defined as in $\S 9$, with modified restrictions on $g_{0}$. We required $g_{0}$ to map each elementary 0 -chain on an elementary 0 -chain and, except when the contrary is stated, $c^{0}$ on $c^{\prime 0}$, where $c^{0}, c^{\prime 0}$ are the elementary 0 -chains associated with the base points. Chain mappings $C(K) \rightarrow C^{*}(L)$, $C^{*}(K) \rightarrow C(L)$, etc., shall satisfy the similar condition, where $C^{*}(K)$, $C^{*}(L)$ are normalized systems determined by $K, L$. Chain homotopy and chain equivalence shall be defined as in $\$ \S 10,11$. We still have (10.2) and (10.3), with these modified definitions. It follows from (10.3) that the homomorphism, $\pi_{1}(K) \rightarrow \pi_{1}(L)$, associated with a chain equivalence, $C(K) \rightarrow C(L)$, is necessarily an isomorphism.

We now prove a very well known theorem, adapted to our set of definitions. Let

$$
g, g^{\prime}: C(K) \rightarrow C(L)
$$

be the chain mappings induced by maps

$$
\phi, \phi^{\prime}:\left(K, e^{0}\right) \rightarrow\left(L, e^{\prime 0}\right) .
$$

Theorem 13. If $\phi \simeq \phi^{\prime}$ then $g \simeq g^{\prime}$.

Let $\phi_{t}: K \rightarrow L$ be a homotopy of $\phi_{0}=\phi$ into $\phi_{1}=\phi^{\prime}$. Let $\tilde{\lambda}: I^{n} \rightarrow \tilde{K}^{n}$ be a map which represents a given element $c \in C_{n}(K)(n \geqq 0)$ and let $\eta_{n+1} c \in C_{n+1}(L)$ be the element represented by $\tilde{\mu}: I^{n+1} \rightarrow \tilde{L}^{n+1}$, where ${ }^{25}$

$$
\tilde{\mu}\left(t, t_{1}, \cdots, t_{n}\right)=\tilde{\phi}_{t} \tilde{\lambda}\left(t_{1}, \cdots, t_{n}\right) .
$$

As in the classical theory of chain homotopy it follows that the map $\eta_{n+1}: C_{n}(K) \rightarrow C_{n+1}(L)$, which is thus defined, is a homomorphism. It follows from (12.4) that it is an operator homomorphism associated

${ }^{25} \tilde{\mu} \partial I^{n+1} \subset \widetilde{L}^{n}$ since $\phi_{t}$, and hence $\tilde{\phi}_{t}$, is cellular. 
with the homomorphism $\bar{f}_{1}: \pi_{1}(K) \rightarrow \pi_{1}(L)$, which is induced by $\phi_{0}$. It follows from (12.1), carried into $\tilde{L}$ by the map $\tilde{\mu}$, and from (12.3) that

$$
\partial_{n+1} \eta_{n+1} c=\left(\bar{w} g_{n}^{\prime}-g_{n}-\eta_{n} \partial_{n}\right) c,
$$

or that $\bar{w} g^{\prime}-g=\partial \eta+\eta \partial$, where $\bar{w}$ means the same as in (12.3). This proves Theorem 13.

THEOREM 14. A map $\phi: K \rightarrow L$ is a homotopy equivalence if, and only if, the induced chain mapping, $g: C(K) \rightarrow C(L)$, is a chain equivalence.

Let $\phi$ be a homotopy equivalence. Then it follows from Theorem 12, by a familiar argument, that $g$ is a chain equivalence.

Conversely, let $g$ be a chain equivalence. Then the associated homomorphism, $\bar{f}_{1}: \pi_{1}(K) \rightarrow \pi_{1}(L)$, which is the one induced by $\phi$, is an isomorphism. Also the induced homomorphism, ${ }^{26} H_{n}(\widetilde{K}) \rightarrow H_{n}(\widetilde{L})$, is an isomorphism, for each $n \geqq 0$. Therefore Theorem 14 follows from Theorem 3 in (I).

Let $K^{0}=e^{0}, L^{0}=e^{\prime 0}$, so that $C(K)=C^{*}(K), C(L)=C^{*}(L)$, and let $f: \rho(K) \rightarrow \rho(L)$ be the homomorphism induced by $\phi: K \rightarrow L$. Then it is to be expected, and is in fact proved in Lemma 9 below, that the chain mapping, $g: C(K) \rightarrow C(L)$, which is induced by $\phi$, is the one induced by $f$. Therefore Theorems 5,6 follow at once from Theorems 13,14 and Theorems 10,12. In general there is a gap between Theorems 13,14 and Theorems 10,12, which we bridge by means of three lemmas concerning $C^{*}(K)$ and $C^{*}(L)$.

The identical map $i: C^{*}(K) \rightarrow C(K)$ is obviously a chain mapping.

Lemma 7. $i: C^{*}(K) \rightarrow C(K)$ is a chain equivalence.

Using the same notation as in the proof of Lemma 6, let $k: C(K)$ $\rightarrow C^{*}(K)$ be the chain mapping which is given by $k \mid C^{*}(K)=1$, $k c_{\lambda}^{\prime}=0, k v_{\lambda}=0$. Then $k i=1$ and $i k-1=\partial \eta+\eta \partial$, where $\eta$ is given by $\eta v_{\lambda}=-c_{\lambda}^{\prime}, \eta c^{0}=0, \eta C_{n}(K)=0$ if $n>0$. This proves the lemma.

Let $i, k$ mean the same as before, both in $K$ and $L$. Let $g, g^{\prime}: C(K)$ $\rightarrow C(L)$ be any chain mappings and let

$$
g^{*}=k g i: C^{*}(K) \rightarrow C^{*}(L), \quad g^{*}=k g^{\prime} i .
$$

Lemma 8. $g^{*} \simeq g^{\prime *}$ if, and only if, $g \simeq g^{\prime}$.

It follows from (13.1) that $g^{*} \simeq g^{\prime *}$ if $g \simeq g^{\prime}$. Let $g^{*} \simeq g^{\prime *}$. Then

$$
g \simeq i k g i k=i g^{*} k \simeq i g^{*} k \simeq g^{\prime}
$$

${ }^{26}$ There is an isomorphism, $H_{n}(\tilde{K}) \approx \partial_{n}^{-1}(0)-\partial_{n+1} C_{n+1}(K)$, which is natural, as the term is used in $\$ 38$ (p. 815) of [9]. 
which proves the lemma.

Let $f: \rho(K) \rightarrow \rho(L)$ be the homomorphism and $g: C(K) \rightarrow C(L)$ the chain mapping induced by a map $\phi: K \rightarrow L$. We shall also describe $g^{*}$, given by (13.1), as induced by $\phi$.

LEMma 9. $g^{*}: C^{*}(K) \rightarrow C^{*}(L)$ is the chain mapping induced by $f: \rho(K) \rightarrow \rho(L)$.

Since $g c^{0}=c^{\prime 0}$ it follows that $g_{0} C_{0}^{*}(K) \subset C_{0}^{*}(L)$. Also $\partial_{1} c^{*} \in C_{0}^{*}(K)$ if $c^{*} \in C_{1}^{*}(K)$. Therefore

$$
\partial_{1} g_{1} c^{*}=g_{0} \partial_{1} c^{*} \in C_{0}^{*}(L)
$$

and it follows from Lemma 6 that $g_{1} C_{1}^{*}(K) \subset C_{1}^{*}(L)$. Since $i$ is the identity and $k \mid C^{*}(L)=1$ it follows that $g^{*}$, in (13.1), is given by $g_{n}{ }^{*} c^{*}=g_{n} c^{*}$ if $c^{*} \in C_{n}{ }^{*}(K)$. Therefore $g_{n} h_{n} a=g_{n}{ }^{*} h_{n} a$ for each $a \in \rho_{n}(K)$ and $n \geqq 1$.

Let $\lambda: I^{n} \rightarrow K^{n}$ be a map representing $a \in \rho_{n}(K)$. Then $f_{n} a$ is represented by $\phi \lambda=\mu$, say, and $h_{n} f_{n} a$ by the lifted map $\tilde{\mu}$. Obviously $\tilde{\mu}=\tilde{\phi} \tilde{\lambda}$, where $\tilde{\phi}, \tilde{\lambda}$ are defined by lifting $\phi, \lambda$. But $\tilde{\phi} \tilde{\lambda}$ represents the element $g_{n} h_{n} a=g_{n}{ }^{*} h_{n} a$. Therefore $h f=g^{*} h$ and the lemma is proved.

Proof of Theorem 5. Let the notation be the same as before and let $\phi \simeq \phi^{\prime}: K \rightarrow L$. Then it follows from Theorem 13 and Lemma 8 that $g^{*} \simeq g^{\prime *}$ and from Lemma 9 and Theorem 10 that $f \simeq f^{\prime}$. This proves Theorem 5.

Proof of Theorem 6. Since $i, k$ are chain equivalences it follows that $g^{*}$, given by (13.1), is a chain equivalence if, and only if, $g$ is a chain equivalence. Therefore Theorem 6 follows from Theorem 14, Lemma 9 and Theorem 12.

As an analogue to the corollary to Theorem 6 we have:

Theorem 15. If $\operatorname{dim} K \leqq m+1$ and if $L$ is a $J_{m}$-complex, then $K \equiv L$ if, and only if, $C(K) \equiv C(L)$. More precisely, any chain equivalence, $C(K) \rightarrow C(L)$, can be realized by a homotopy equivalence, $K \rightarrow L$, and conversely.

Since $k: C(K) \rightarrow C^{*}(K)$ and $i: C^{*}(L) \rightarrow C(L)$ are chain equivalences this follows from the corollary to Theorem 6, from Lemma 9 and from Theorem 12.

We conclude the section with a theorem on the realizability of chain mappings, which is analogous to Theorem 4. Let $g: C(K) \rightarrow C(L)$ be a chain mapping associated with a homomorphism $f: \pi_{1}(K) \rightarrow \pi_{1}(L)$. Let $K_{0} \subset K$ be a connected sub-complex, which contains $e^{0}$. Let

$$
i: \pi_{1}\left(K_{0}\right) \rightarrow \pi_{1}(K), \quad j: C\left(K_{0}\right) \rightarrow C(K)
$$


be the homomorphism and the chain mapping, associated with $\bar{i}$, which are induced by the identical map $K_{0} \rightarrow K$. We describe a map, $\phi_{0}: K_{0} \rightarrow L$, as a partial realization of $g$ if, and only if, it induces the chain mapping

$$
g j: C\left(K_{0}\right) \rightarrow C(L) .
$$

The chain mapping $g j$ is associated with $\bar{f}^{0}=\bar{f} \bar{\imath}: \pi_{1}\left(K_{0}\right) \rightarrow \pi_{1}(L)$. Therefore, if $\phi_{0}$ induces $g j$, the homomorphism $\bar{f}^{0}$ is the one induced ${ }^{27}$ by $\phi_{0}$.

Let $\phi_{0}: K_{0} \rightarrow L$ be a partial realization of $g$. Let $\bar{K}_{0}$, with a base point covering $e^{0}$, be a universal covering space of $K_{0}$. Then $g j$ is the chain mapping induced by the lifted map $\bar{\phi}_{0}: \bar{K}_{0} \rightarrow \widetilde{L}$. Let $\widetilde{K}_{0} \subset \widetilde{K}$ be the component of $p^{-1} K_{0}$ which contains $\tilde{e}^{0}$. Then $\tilde{K}_{0}$ is a covering complex of $K_{0}$, associated with the sub-group $i^{-1}(1) \subset \pi_{1}\left(K_{0}\right)$, and $\bar{K}_{0}$ is a universal covering complex of $\widetilde{K}_{0}$, with a covering map $\bar{p}: \bar{K}_{0} \rightarrow \widetilde{K}_{0}$. Since $\bar{f}^{0} \bar{\imath}^{-1}(1)=\bar{f} \bar{\imath} \bar{\imath}^{-1}(1)=1$ the map $\phi_{0}$ can be lifted ${ }^{23}$ into a map $\tilde{\phi}_{0}: \widetilde{K}_{0} \rightarrow \tilde{L}$, and $\bar{\phi}_{0}$ is the map obtained by lifting $\tilde{\phi}_{0}$ into $\bar{K}$. Therefore

$$
\bar{\phi}_{0}=\tilde{\phi}_{0} \bar{p}: \bar{K}_{0} \rightarrow \tilde{L}
$$

and we may regard $g j$ as the chain mapping which is induced by $\phi_{0}$. We state this, in a slightly different form, as:

Lemma 10. If $c \in C_{n}(K)$ has a representative map, $\tilde{\lambda}: I^{n} \rightarrow \tilde{K}_{0}^{n}$, then $g_{n} c$ is represented by the map $\tilde{\phi}_{0} \tilde{\lambda}: I^{n} \rightarrow \tilde{L}^{n}$.

THeOREM 16. If $K_{0} \cup K^{m+1}=K$ and if $L$ is a $J_{m}$-complex, then any partial realization, $\phi_{0}: K_{0} \rightarrow L$, of a given chain mapping, $g: C(K) \rightarrow C(L)$, can be extended to a full realization, $\phi: K \rightarrow L$.

First assume that $K^{1} \subset K_{0}$. Then $K_{0}^{1}=K^{1}, \tilde{K}_{0}^{1}=\tilde{K}^{1}$ and $^{28} \rho_{1}\left(K_{0}\right)$ $=\rho_{1}(K)$. Let $f^{0}: \rho\left(K_{0}\right) \rightarrow \rho(L)$ be the homomorphism induced by $\phi_{0}$ and let $g^{*}: C^{*}(K) \rightarrow C^{*}(L)$ mean the same as in (13.1). Let

$$
\lambda:(I, 0,1) \rightarrow\left(K^{1}, e^{0}, e^{0}\right)
$$

be a map representing a given element $x \in \rho_{1}(K)$ and let $\tilde{\lambda}: I \rightarrow \tilde{K}^{1}=\tilde{K}_{0}^{1}$ be the lifted map. Then it follows from Lemma 10 that $g_{1}^{*} h_{1} x=g_{1} h_{1} x$ is represented by the map $\tilde{\phi}_{0} \tilde{\lambda}$, which also represents $h_{1} f_{1}^{0} x$. Therefore $h_{1} f_{1}^{0}=g_{1}^{*} h_{1}$. Hence it follows from the addendum to Theorem 9 that there is a realization, $f: \rho(K) \rightarrow \rho(L)$, of $g^{*}$, such that $f_{1}=f_{1}^{0}$.

I say that $\phi_{0}$ is a partial realization of $f$, or that $f^{0}=f i$, where $i: \rho\left(K_{0}\right)$ $\rightarrow \rho(K)$ is the injection homomorphism. For, by Lemma $9, i$ is a real-

${ }^{27} \mathrm{Cf}$. a remark following (2.2).

${ }^{28}$ In general $C_{1}\left(K_{0}\right) \neq C_{1}(K)$ since $C_{1}\left(K_{0}\right)$ refers to $\bar{K}_{0}$. 
ization of the chain mapping, $j^{*}: C^{*}\left(K_{0}\right) \rightarrow C^{*}(K)$, which is induced by the identical map $K_{0} \rightarrow K$. Therefore $f i$ is a realization of $g^{*} j^{*}$. Since $g^{*}$ is also given by $g^{*} c^{*}=g c^{*}\left(c^{*} \in C^{*}(K)\right)$ it follows that $g^{*} j^{*}=(g j)^{*}$. But $(g j)^{*}$ is induced by $\phi_{0}$ and therefore by $f^{0}$, according to Lemma 9. Therefore both $f i$ and $f^{0}$ are realizations of $(g j)^{*}$. Since $i_{1}=1$ we have $(f i)_{1}=f_{1}=f_{1}^{0}$. Therefore $f^{0}=f i$, by the addendum to Theorem 9. Hence it follows from Theorem 4 that $\phi_{0}$ can be extended to a map, $\phi: K \rightarrow L$, which realizes $f$ and hence $g^{*}$. Let $\widetilde{\phi}$ be the lifted map. Then $\tilde{\phi} \mid \widetilde{K}_{0}=\widetilde{\phi}_{0}$, since $\phi \mid K_{0}=\phi_{0}$. Since $\widetilde{K}_{0}^{1}=\widetilde{K}^{1}$ it follows from Lemma 10 that $\widetilde{\phi}$ induces the homomorphisms $g_{0}, g_{1}$. Therefore $\phi$ induces $g$. Thus the theorem is proved on the assumption that $K^{1} \subset K_{0}$.

In general let $K_{1}=K_{0} \cup K^{1}$. The theorem will follow from what we have already proved when we have extended $\phi_{0}$ to a partial realization, $\phi_{1}: K_{1} \rightarrow L$, of $g$. We first extend $\widetilde{\phi}_{0}: \widetilde{K}_{0} \rightarrow \tilde{L}$ to a map $\psi: p^{-1} K_{0} \rightarrow \tilde{L}$ by writing

$$
\psi \tau(\bar{x}) p=\tau(\bar{f} \bar{x}) \tilde{\phi}_{0} p \quad\left(p \in \tilde{K}_{0}\right),
$$

for every $\bar{x} \in \pi_{1}(K)$. We then extend $\psi$ to a map

$$
\psi^{\prime}:\left(p^{-1} \tilde{K}_{0}\right)=\tilde{K}^{0} \rightarrow \tilde{L},
$$

which induces $g_{0}$. This is possible since $g_{0}$ maps elementary 0 -chains into elementary 0 -chains. Moreover

$$
\psi^{\prime} \tau(x) \cup \tau(\bar{f} \bar{x}) \psi^{\prime},
$$

since $g_{0} \bar{x}=(\bar{f} \bar{x}) g_{0}$.

Let $e^{1}$ be any 1 -cell in $K^{1}-K_{0}$. Let $\tilde{e}^{1} \in \tilde{K}^{1}$ be a 1 -cell which covers $e^{1}$ and let $c^{1} \in C_{1}(K)$ be an elementary 1-chain associated with $\tilde{e}^{1}$. Then $\partial c^{1}=v-u$, where $u, v \subset C_{0}(K)$ are the elementary 0 -chains associated with the end points, $p, q \subset \widetilde{K}^{0}$, of $\tilde{e}^{1}$. Also

$$
\partial g_{1} c^{1}=g_{0} \partial_{1} c^{1}=g_{0} v-g_{0} u
$$

and $g_{0} u, g_{0} v$ are the elementary 0 -chains associated with $\psi^{\prime} p, \psi^{\prime} q$. Therefore it follows from an argument similar to the proof of Theorem 8 (c) that $g_{1} c^{1}$ is represented by a map

$$
\mu:(I, 0,1) \rightarrow\left(\tilde{L}^{1}, \psi^{\prime} p, \psi^{\prime} q\right) .
$$

If $p=q$ then $\psi^{\prime} p=\psi^{\prime} q$, and it follows that the map

$$
\theta_{e^{1}: \mu \lambda^{-1}: \bar{e}^{1}} \rightarrow \widetilde{L}^{1}
$$

is single-valued and continuous, where $\lambda: I \rightarrow \overline{\tilde{e}}^{1}$ is a characteristic map for $\tilde{e}^{1}$. Therefore $\psi^{\prime}$ may be extended to a map, $\tilde{\phi}_{1}: p^{-1} K_{1} \rightarrow \tilde{L}$, by writing 


$$
\widetilde{\phi}_{1} \mid \tau(\bar{x}) \overline{\tilde{e}^{1}}=\tau(\bar{f} \bar{x}) \theta_{e^{1}},
$$

for every 1-cell $e^{1} \in K^{1}-K_{0}$ and every $\bar{x} \in \pi_{1}(K)$. Then $\tilde{\phi}_{1}$ induces $g_{0}$ and $g_{1}$. Clearly $\tilde{\phi}_{1} \tau(\bar{x})=\tau(\bar{f} \bar{x}) \widetilde{\phi}_{1}$ and the required map, $\phi_{1}: K \rightarrow L$, is defined by $\phi_{1} p=p \tilde{\phi}_{1}$. This completes the proof.

14. Proof of Theorem 7. Let $P=K \times I$. Then $\tilde{P}=\tilde{K} \times I$ is a universal covering complex of $P$ and we take $\left(e^{0}, 0\right)$ and $\left(\tilde{e}^{0}, 0\right)$ as base points in $P$ and $\tilde{P}$. The $n$-cells in $\tilde{P}$ are the cells $\widetilde{e}^{n} \times 0, \widetilde{e}^{n} \times 1, \widetilde{e}^{n-1} \times(0$, 1) for all $n$-cells and $(n-1)$-cells in $\tilde{K}$. Let $\tilde{\theta}_{t}: \tilde{K} \rightarrow \widetilde{P}$ be the homotopy, which is given by $\tilde{\theta}_{t} \tilde{q}=(\tilde{q}, t)(\tilde{q} \in \tilde{K})$. Let $\alpha^{0}, \alpha^{1}: C(K) \rightarrow C(P)$ be the chain mappings ${ }^{29}$ induced by $\tilde{\theta}_{0}, \tilde{\theta}_{1}$ and let

$$
\gamma: C_{n}(K) \rightarrow C_{n+1}(P)
$$

be the chain deformation operator which is defined as in Theorem 13, with $\tilde{L}=\widetilde{P}, \tilde{\phi}_{t}=\tilde{\theta}_{t}$. Then $\left(\alpha^{0} c_{\lambda}^{n}, \alpha^{1} c_{\lambda}^{n}, \gamma c_{\mu}^{n-1}\right)$ is obviously a basis for $C_{n}(P)$, where $\left\{c_{\lambda}^{n}\right\},\left\{c_{\mu}^{n-1}\right\}$ are bases for $C_{n}(K), C_{n-1}(K)$. The operator $\partial: C_{n}(P) \rightarrow C_{n-1}(P)$ is defined by

$$
\begin{aligned}
\partial \alpha^{\nu} c_{\lambda}^{n} & =\alpha^{\nu} \partial c_{\lambda}^{n} \\
\partial \gamma c_{\mu}^{n-1} & =\left(\alpha^{1}-\alpha^{0}-\gamma \partial\right) c_{\mu}^{n-1},
\end{aligned} \quad(\nu=0,1)
$$

which are the same as $\partial \alpha^{\nu}=\alpha^{\nu} \partial, \alpha^{1}-\alpha^{0}=\partial \gamma+\gamma \partial$.

Let $g^{\nu}: C(K) \rightarrow C(L)(\nu=0,1)$ be chain mappings, which are related by

$$
\bar{w} g^{1}-g^{0}=\partial \eta+\eta \partial
$$

where $\eta: C(K) \rightarrow C(L)$ is a chain deformation operator and $\bar{w} \in \pi_{1}(L)$. Then it follows from (14.1) and (14.2) that $g: C(P) \rightarrow C(L)$ is a chain mapping, where $g$ is defined by

$$
g \alpha^{0}=g^{0}, \quad g \alpha^{1}=\bar{w} g^{1}, \quad g \gamma=\eta .
$$

Conversely (14.2) follows from (14.1), (14.3) and $g \partial=\partial g$.

Now let $g^{\nu}$ have a geometrical realization, $\phi^{\nu}: K \rightarrow L$, let $g^{0}, g^{1}$ be related by $(14.2)$ and let

$$
\psi:(K \times 0) \cup\left(e^{0} \times I\right) \cup(K \times 1) \rightarrow L
$$

be given by $\psi(q, \nu)=\phi^{\nu} q(q \in K), \psi\left(e^{0}, t\right)=\lambda(t)$, where $\lambda: I \rightarrow L^{1}$ represents $\bar{v}$. Then it is easily verified that $\psi$ is a partial realization of $g: C(P) \rightarrow C(L)$. If $\operatorname{dim} K \leqq m$, whence $\operatorname{dim} P \leqq m+1$, and if $L$ is a

\footnotetext{
${ }^{29}$ N.B. $\alpha^{1} c^{0} \neq c^{\prime 0}$, where $c^{0}, c^{\prime 0}$ are the elementary 0 -chains associated with the base points $\tilde{e}^{0} \in \tilde{K}^{0}$ and $\left(\tilde{e}^{0}, 0\right)$.
} 
$J_{m}$-complex it follows from Theorem 16 that $\psi$ can be extended throughout $P$, whence $\phi^{0} \simeq \phi^{1}$. Thus $\phi^{0} \simeq \phi^{1}$ if $g^{0} \simeq g^{1}$.

Theorem 7 now follows from Lemmas 8, 9 and Theorem 10.

15. Examples. Let $P$ and $Q$ be 3 -dimensional lens spaces (see $[14$, pp. 210,279$])$ of types $(m, p)$ and $(m, q)$ where $m$ is the order of $\pi_{1}(P)$ and $\pi_{1}(Q)$. It was proved (see also [15]) in [3] that, if there is an integer, $k$, such that $q \equiv \pm k^{2} p(m)$, then $P \equiv Q$. We give an alternative proof of this by exhibiting a chain equivalence $u: C(P) \rightarrow C(Q)$.

As usual we suppose that $P, Q$ are complexes such that $C_{n}(P)$, $C_{n}(Q)$ have bases consisting of single elementary $n$-chains, ${ }^{30} a_{n}, b_{n}$ $(n=0, \cdots, 3)$, and that

$$
\begin{aligned}
\partial a_{1}=(\xi-1) a_{0}, & \partial a_{2}=\sigma_{m}(\xi) a_{1}, & \partial a_{3}=\left(\xi^{p}-1\right) a_{2}, \\
\partial b_{1}=(\eta-1) b_{0}, & \partial b_{2}=\sigma_{m}(\eta) b_{1}, & \partial b_{3}=\left(\eta^{q}-1\right) b_{2},
\end{aligned}
$$

where $\xi, \eta$ generate $\pi_{1}(P), \pi_{1}(Q)$ and

$$
\sigma_{t}\left(\zeta^{s}\right)=1+\zeta^{s}+\cdots+\zeta^{(t-1) s} \quad(\zeta=\xi \text { or } \eta) .
$$

Let $q \equiv-k^{2} p(m)$. Then we replace $b_{3}$ by $-b_{3}$ and $b_{2}$ by $\eta^{q} b_{2}$. Since $\eta^{q} \sigma_{m}(\eta)=\sigma_{m}(\eta)$ we have

$$
\partial\left(-b_{3}\right)=\left(\eta^{-q}-1\right) \eta^{q} b_{2}, \quad \partial\left(\eta^{q} b_{2}\right)=\sigma_{m}(\eta) b_{1} .
$$

Therefore this has the effect of replacing $q$ by $-q$ and we assume that $q \equiv k^{2} p$. Since $(p, m)=(q, m)=1$ we have $(k, m)=1$ and there are integers, $h, l$, such that $k l=1+h m$. Then $p \equiv l^{2} q(m)$.

Let

$$
u: C(P) \rightarrow C(Q), \quad v: C(Q) \rightarrow C(P)
$$

be the chain mappings, which are associated with the mutually inverse isomorphisms $\xi \rightarrow \eta^{k}, \eta \rightarrow \xi^{l}$, and are given by $u a_{n}=b_{n}, v b_{n}=a_{n}$ if $n=0$ or 3 and

$$
\begin{aligned}
u a_{1}=\sigma_{k}(\eta) b_{1}, & u a_{2}=\sigma_{k}\left(\eta^{k p}\right) b_{2}, \\
v b_{1}=\sigma_{l}(\xi) a_{1}, & v b_{2}=\sigma_{l}\left(\xi^{l q}\right) a_{2} .
\end{aligned}
$$

It is easily verified that $\partial u=u \partial, \partial v=v \partial$.

I say that, if $s, t$ are integers such that $l t \equiv s(m)$, then

$$
\sigma_{k}\left(\xi^{s}\right) \sigma_{l}\left(\xi^{t}\right)=1+h \sigma_{m}(\xi) .
$$

For let $\omega_{0}=1, \omega_{1}, \cdots, \omega_{m-1}$ be the $m$ th roots of unity and let $\chi_{r}$ be the character of $\pi_{1}(P)$, which is given by $\chi_{r}(\xi)=\omega_{r}$. It follows from the

${ }^{30}$ In these examples the dimensionality of a chain is indicated by a subscript. 
orthogonality relations,

$$
\sum_{r=0}^{m-1} \bar{\chi}_{r}\left(\xi^{i}\right) \chi_{r}\left(\xi^{j}\right)=m \delta^{i j} \quad(i, j=0, \cdots, m-1),
$$

that $\lambda=0$ if $\chi_{r}(\lambda)=0$ for every $r=0, \cdots, m-1$, where $\lambda$ is in the group ring of $\pi_{1}(P)$. But

$$
\chi_{0}\left\{\sigma_{l}\left(\xi^{s}\right) \sigma_{l}\left(\xi^{t}\right)\right\}=k l=1+h m=\bar{\chi}_{0}\left\{1+h \sigma_{m}(\xi)\right\} .
$$

Also $k s \equiv k l t \equiv t(m)$ and $\chi_{r}\left\{\sigma_{m}(\xi)\right\}=0$ if $r \neq 0$, whence

$$
\begin{aligned}
\chi_{r}\left\{\sigma_{k}\left(\xi^{s}\right) \sigma_{l}\left(\xi^{t}\right)\right\} & =\frac{\omega_{r}^{l s}-1}{\omega_{r}^{s}-1} \cdot \frac{\omega_{r}^{l t}-1}{\omega_{r}^{t}-1}=1 \\
& =\bar{\chi}_{r}\left\{1+h \sigma_{m}(\xi)\right\},
\end{aligned}
$$

which proves (15.3).

It follows from (15.2) and (15.3), first with $s=l, t=1$ and secondly with $s=k l p \equiv p, t=l q$, that

$$
\begin{aligned}
v u a_{1} & =v \sigma_{k}(\eta) b_{1}=\sigma_{k}\left(\xi^{l}\right) \sigma_{l}(\xi) a_{1} \\
& =\left\{1+h \sigma_{m}(\xi)\right\} a_{1}, \\
v u a_{2} & =v \sigma_{k}\left(\eta^{k p}\right) b_{2}=\sigma_{k}\left(\xi^{k l p}\right) \sigma_{l}\left(\xi^{l q}\right) a_{2} \\
& =\left\{1+h \sigma_{m}(\xi)\right\} a_{2} .
\end{aligned}
$$

Therefore $v u-1=\partial \delta+\delta \partial$, where $\delta a_{0}=0, \delta a_{1}=h a_{2}, \delta a_{2}=0$, whence $v u \simeq 1$. Similarly $u v \simeq 1$ and it follows from Theorem 15 that $u$ can be realized by a homotopy equivalence $P \rightarrow Q$.

Our next example is of a 5-dimensional homotopy system, $\rho=\left\{\rho_{n}\right\}$ which has no geometrical realization. It is defined as follows.

(a) $\rho_{1}$ has a single basis element $x$,

(b) $\rho_{2}$ has two basis elements, $a_{2}, b_{2}$ and $d a_{2}=x^{2}, d b_{2}=1$,

(c) $\rho_{n}(n=3,4,5)$ has a single basis element, $a_{n}$,

(d) $d a_{3}=(\xi-1) b_{2}, d a_{4}=(\xi+1) a_{3}, d a_{5}=(\xi-1) a_{4}$, where $\xi=\bar{x}$.

Then $d d=0$ since $\xi^{2}=1$. We shall prove that $\rho$ has no geometrical realization.

We can realize the system $\left(\rho_{1}, \cdots, \rho_{4}, 0, \cdots\right)$ by the topological product $Q^{4}=P^{2} \times S^{2}$, where $P^{2}=e_{0}^{0} \cup e_{0}^{1} \cup e_{0}^{2}$ and $S^{2}=e_{1}^{0} \cup e_{1}^{2}$ are complexes covering a real projective plane and a 2 -sphere respectively. Assume that $\rho$ has a realization $K_{0}$. Then $Q^{3}$ and $K_{0}^{3}$ are two realizations of the system $\rho^{3}=\left(\rho_{1}, \rho_{2}, \rho_{3}, 0, \cdots\right)$. By Theorem 4 there is a map $\phi: K_{0}^{3} \rightarrow Q^{3}$, which realizes the resultant, $\rho\left(K_{0}^{3}\right) \rightarrow \rho\left(Q^{3}\right)$, of given isomorphisms $\rho\left(K_{0}^{3}\right) \rightarrow \rho^{3}$ and $\rho^{3} \rightarrow \rho\left(Q^{3}\right)$. Let $K$ be the complex formed by identifying each point $p \in K_{0}^{3}$ with $\phi p \in Q^{3}$. Then $K^{3}=Q^{3}$ and the 
map $\psi: K_{0} \rightarrow K$, which is given by $\psi\left|K_{0}^{3}=\phi, \psi\right| K_{0}-K_{0}^{3}=1$, obviously induces an isomorphism $\rho\left(K_{0}\right) \rightarrow \rho(K)$. Therefore $K$ is another realization of $\rho$. Let us take $\rho=\rho(K)$. It is easily verified that any basis for $\rho_{n}(n=4,5)$ must consist of a single element, which is of the form $\pm \xi^{r} a_{n}(r=0$ or 1$)$. Therefore $K$ has but a single $n$-cell, $e^{n}$. Let $a_{n}$ be represented by a characteristic map for $e^{n}$. We assume, as we obviously may, that $a_{2}, b_{2}, a_{3}$ are represented by characteristic maps for cells in $Q^{3}$. Since $(\xi-1) a_{4}=d a_{5}=j \beta a_{5}$, where $j, \beta$ mean the same as in $\S 5$, and since $\beta j=0$ we have

$$
(\xi-1) \beta a_{4}=\beta(\xi-1) a_{4}=0 .
$$

It follows from the condition (b) that $d_{2}^{-1}(1)$, in $\rho_{2}$, is a free Abelian group, which is freely generated by $(\xi-1) a_{2}, b_{2}, \xi b_{2}$. Moreover $j: \pi_{2}\left(Q^{2}\right)$ $\rightarrow \rho_{2}$ is an isomorphism onto $d_{2}^{-1}(1)$ and we identify each $a \in \pi_{2}\left(Q^{2}\right)$ with $j a \in \rho_{2}$. Let $\tilde{P}^{2}$ be a universal covering complex of $P^{2}$. Then $\widetilde{Q}^{4}=\widetilde{P}^{2} \times S^{2}$, with a base point, $\widetilde{e}^{0}=\left(\widetilde{e}_{0}^{0}, e_{1}^{0}\right)$ covering $\left(e_{0}^{0}, e_{1}^{0}\right)$, is a universal covering complex of $Q^{4}$. We have

$$
\tilde{Q}^{2}=S_{0}^{2} \times S_{1}^{2} \times \tau(\xi) S_{1}^{2},
$$

where $\tau(\xi)$ means the same as in $\$ 12$ and $S_{0}^{2}=\widetilde{P}^{2} \times \widetilde{e}_{1}^{0}, S_{1}^{2}=\widetilde{e}_{0}^{0} \times S^{2}$ and $S_{1}^{2}$ and $\tau(\xi) S_{1}^{2}$ are attached to $S_{0}^{2}$ at the points $\tilde{e}^{0}$ and $\tau(\xi) \tilde{e}^{0}$. The elements $(\xi-1) a_{2}, b_{2}, \xi b_{2}$ are represented by homomorphisms

$$
\partial I^{3} \rightarrow S_{0}^{2}, \quad \lambda: \partial I^{3} \rightarrow S_{1}^{2}, \quad \tau(\xi) \lambda: \partial I^{3} \rightarrow \tau(\xi) S_{1}^{2},
$$

projected into $Q^{2}$ by the covering map $p: \widetilde{Q}^{4} \rightarrow Q^{4}$. Let $\gamma$ be a generator of $\pi_{3}\left(\partial I^{3}\right)$ and, if $a \in \pi_{2}(X)$, where $X$ is any space, let $a \cdot \gamma \in \pi_{3}(X)$ be the element respresented by the resultant of a map $\partial I^{4} \rightarrow \partial I^{3}$, representing $\gamma$, followed by a map $\partial I^{3} \rightarrow X$, representing $a$. Then it follows from an easy extension of Theorem 2 in [2], applied to $\widetilde{Q}^{2}$ and transferred to $Q^{2}$ by means of $p: \widetilde{Q}^{4} \rightarrow Q^{4}$, that $\pi_{3}\left(Q^{2}\right)$ is a free Abelian group, which is freely generated by

$$
u=\left(\xi a_{2}-a_{2}\right) \cdot \gamma, \quad v=b_{2} \cdot \gamma, \quad\left(\xi b_{2}\right) \cdot \gamma,
$$

together with

$$
\left[(\xi-1) a_{2}, b_{2}\right], \quad\left[(\xi-1) a_{2}, \xi b_{2}\right], \quad\left[b_{2}, \xi b_{2}\right],
$$

where $[a, b]$ means the same as $a \cdot b$ in [2]. Obviously $\xi(a \cdot \gamma)=(\xi a) \cdot \gamma$. Also $^{31}(-\tilde{a}) \cdot \gamma=\tilde{a} \cdot \gamma$ if $\tilde{a} \in \pi_{2}\left(S_{0}^{2}\right)$. Therefore

$$
\xi u=\left(a_{2}-\xi a_{2}\right) \cdot \gamma=\left(\xi a_{2}-a_{2}\right) \cdot \gamma=u .
$$

${ }^{31}$ See Theorem IIb' on p. 639 of [16]. 
Let $i$ denote both injection homomorphisms $i: \pi_{n}\left(Q^{2}\right) \rightarrow \pi_{n}\left(Q^{3}\right)$ $(n=2,3)$. Since $j_{2}=1$ we have

$$
\beta a_{3}=d a_{3}=(\xi-1) b_{2} .
$$

Therefore $i \xi b_{2}=i b_{2}, i \xi v=\left(i \xi b_{2}\right) \quad \gamma=i v$ and

$$
i\left[a, \xi b_{2}\right]=\left[i a, i \xi b_{2}\right]=\left[i a, i b_{2}\right]=i\left[a, b_{2}\right],
$$

for any $a \in \pi_{2}\left(Q^{2}\right)$. Also a calculation of the Hopf invariant shows that $\left[b_{2}, b_{2}\right]= \pm 2 v$. Therefore $i \pi_{3}\left(Q^{2}\right)$ is generated by $\bar{u}, \bar{v}, w \bar{w}$, where

$$
\bar{u}=i u, \quad \bar{v}=i v, \quad \bar{w}=i w, \quad w=\left[(\xi-1) a_{2}, b_{2}\right] .
$$

Notice that $\xi \bar{u}=i \xi u=\bar{u}, \xi \bar{v}=\bar{v}$.

Let $b_{4} \in \rho_{4}\left(Q^{4}\right)$ be the element which is represented by a characteristic map for $e_{0}^{2} \times e_{1}^{2}$, in the sense class which makes $d b_{4}=d a_{4}=(\xi+1) a_{3}$. Let $e^{\prime 2}=\widetilde{P}^{2}-\widetilde{e}_{0}^{0}$. Then

$$
S^{\prime 2}=\tilde{e}_{0}^{0} \cup e^{\prime 2}, \quad Q^{\prime 4}=S^{\prime 2} \times S^{2}
$$

are complexes of which $\widetilde{P}^{2}, \widetilde{Q}^{4}$ are sub-divisions. Let $a_{2}^{\prime}, b_{2}^{\prime} \subset \rho_{2}\left(Q^{\prime 2}\right)$ $b_{4}^{\prime} \in \rho_{4}\left(Q^{\prime 4}\right)$ be the elements represented by characteristic maps for $e^{\prime 2} \times e_{1}^{0}, \widetilde{e}_{0}^{0} \times e_{1}^{2}$ and $e^{\prime 2} \times e_{1}^{2}$. Then it follows from the definition of $[a, b]$ that $\beta b_{4}^{\prime}= \pm\left[a_{2}^{\prime}, b_{2}^{\prime}\right]$. Also the homomorphism $\rho\left(Q^{\prime 4}\right) \rightarrow \rho\left(Q^{4}\right)$, which is induced by the identical map $Q^{\prime 4} \rightarrow \widetilde{Q}^{4}$, followed by $p: \widetilde{Q}^{4} \rightarrow Q^{4}$, carries $a_{2}^{\prime}, b_{2}^{\prime}, b_{4}^{\prime}$ into $\pm(\xi-1) a_{2}, \pm b_{2}, \pm(\xi-1) b_{4}$. Therefore $\beta(\xi-1) b_{4}$ $= \pm \bar{w}$.

I say that $\bar{w}$ is not of finite order. For

$$
\pi_{4}\left(Q^{4}\right) \approx \pi_{4}\left(S^{\prime 2}\right)+\pi_{4}\left(S^{2}\right),
$$

whence $\pi_{4}\left(Q^{4}\right)$ is of order 4. Since $\rho_{4}\left(Q^{4}\right)$ is a free Abelian group it follows that $j \pi_{4}\left(Q^{4}\right)=0$. Therefore it follows from the exactness of the sequence

$$
\pi_{4}\left(Q^{4}\right) \stackrel{j}{\rightarrow} \rho_{4}\left(Q^{4}\right) \stackrel{\beta}{\rightarrow} \pi_{3}\left(Q^{3}\right)
$$

that $\beta$ is an isomorphism into $\pi_{3}\left(Q^{3}\right)$. Since $(\xi-1) b_{4}$ is not of finite order neither is $\bar{w}$.

We now contradict (15.4). Since $j\left(\beta a_{4}-\beta b_{4}\right)=d a_{4}-d b_{4}=0$ it follows that $\beta a_{4}-\beta b_{4} \in i \pi_{3}\left(Q^{2}\right)$. Therefore

$$
\begin{aligned}
\beta a_{4} & =\beta b_{4}+l \bar{u}+m \bar{v}+n \bar{v} \\
& =\beta b_{4}+l \bar{u}+m \bar{v} \pm n \beta(\xi-1) b_{4} \\
& =\{1 \pm n(\xi-1)\} \beta b_{4}+l \bar{u}+m \bar{v},
\end{aligned}
$$

where $l, m, n$ are integers. Since $\xi^{2}=1$ we have $(\xi-1)^{2}=2(\xi-1)$ and 


$$
(\xi-1)\{1 \pm n(\xi-1)\}=(1 \pm 2 n)(\xi-1) .
$$

Also $(\xi-1) \bar{u}=(\xi-1) \bar{v}=0$. Therefore

$$
(\xi-1) \beta a_{4}=(1 \pm 2 n)(\xi-1) \beta b_{4}=(1 \pm 2 n) \bar{w} \neq 0 .
$$

Therefore it is absurd to suppose that $\rho$ has a geometrical realization.

The above argument serves as an example of the use of the groups $\pi_{n}\left(K^{n}\right)$ and $j^{-1}(0)=i \pi_{n}\left(K^{n-1}\right)$, which is discussed in $\$ 17$ below.

16. Note on $\rho_{n}(K)$. Let $\rho=\rho(K)$, where $K$ is a given complex. According to (D) in $\S 5$ of (I) any compact sub-set of $K$ is contained in a finite sub-complex of $K$. Therefore the fact that $\rho_{n}$ is a free $\bar{\rho}_{1-}$ module if $n>2$ follows from arguments on p. 417 of [2], restated in terms of relative homotopy groups. Alternatively one may prove the result for $H_{n}\left(\tilde{K}^{n}, \tilde{K}^{n-1}\right)$, using the Eilenberg-Steenrod "excision" Theorem, where $\tilde{K}$ is a universal covering complex of $K$.

We now show how the arguments on pp. 422-425 of [2] can be used to prove that $\rho_{2}$ is a free crossed $\left(\rho_{1}, d_{2}\right)$-module. More generally let

$$
X=X_{0} \cup\left\{e_{\lambda}^{2}\right\}
$$

where the cells $\left\{e_{\lambda}^{2}\right\}$ are attached to $X_{0}$ as in [2]. We allow this set of cells to be infinite provided $X$ is a Hausdorff space such that

(a) $X_{0}$ is an arcwise connected, closed sub-set of $X$, and

(b) $Y \subset X$ is closed if $Y \cap X_{0}$ and all the sets $Y \cap \bar{e}_{\lambda}^{2}$ are closed.

Let this be so. Then it follows from the proof of (D) in $\$ 5$ of (I) that any compact sub-set of $X$ lies in the union of $X_{0}$ and a finite subset of the cells $\left\{e_{\lambda}^{2}\right\}$.

Let $\rho_{2}=\pi_{2}\left(X, X_{0}\right), \rho_{1}=\pi_{1}\left(X_{0}\right)$, with a common base point $x_{0} \in X_{0}$, and let $d: \rho_{2} \rightarrow \rho_{1}$ be the boundary homomorphism. Let $a_{\lambda}^{\prime} \in \rho_{2}$ be the element which is defined by a characteristic map ${ }^{32}$ for $e_{\lambda}^{2}$, joined to $x_{0}$ by a path in $X_{0}$. We define a free crossed $\left(\rho_{1}, \phi\right)$-module $h_{\pi}\left(=h_{\pi_{1}}\right.$ in [2]), with a basis, $\left\{a_{\lambda}\right\}$, and with $\phi a_{\lambda}=d a_{\lambda}^{\prime}$. It follows from Lemma 2 , in $\$ 2$ above, that the correspondence $a_{\lambda} \rightarrow a_{\lambda}^{\prime}$ determines an operator homomorphism, $\theta: h_{\pi} \rightarrow \rho_{2}$, such that $\phi=d \theta$. Obviously $\theta$ is onto $\rho_{2}$ and we shall prove that it is an isomorphism.

Let $\sigma_{\lambda}^{2} \subset e_{\lambda}^{2}$ be a 2 -simplex. Then we may absorb the closure of $e_{\lambda}^{2}-\sigma_{\lambda}^{2}$ into $X_{0}$, for each $\lambda$. In order to simplify the notation we start again with $e_{\lambda}^{2}=\sigma_{\lambda}^{2}-\partial \sigma_{\lambda}^{2}$ and $\sigma_{\lambda}^{2} \subset e_{\lambda}^{\prime 2}$, where $e_{\lambda}^{\prime 2}$ is a 2-cell, which is an open sub-set of $X$. Let $p_{\lambda}$ be a vertex of $\sigma_{\lambda}^{2}$. Any element of $\rho_{2}$ may be

${ }^{32}$ I.e. a map, $\psi:\left(I^{2}, \partial I^{2}\right) \rightarrow\left(X_{0} \cup e_{\lambda}^{2}, X_{0}\right)$, such that $\psi \mid I^{2}-\partial I^{2}$ is a homeomorphism onto $e_{\lambda}^{2}$. 
represented by a map,

$$
f:\left(I^{2}, \partial I^{2}, I^{0}\right) \rightarrow\left(X, X_{0}, x_{0}\right),
$$

which is normal in the following sense. The closure of $f^{-1} e_{\lambda}^{2}$ is a (finite) set of rectilinear simplexes, $\sigma_{1 \lambda}^{2}, \sigma_{2 \lambda}^{2}, \cdots \subset I^{2}-\partial I^{2}$, such that each $f \mid \sigma_{i \lambda}^{2}$ is a barycentric map on $\sigma_{\lambda}^{2}$. Let $\left\{s_{i \lambda}\right\}$ be a set of polygonal segments in $I^{2}$, which join $I^{0}$ to the vertices $p_{i \lambda}=f^{-1} p_{\lambda} \cap \sigma_{i \lambda}^{2}$ and do not meet each other or the simplexes $\sigma_{j \mu}^{2}$, except in the end points $I^{0}$, $p_{i \lambda}$. For reasons given on p. 422 of [2], a normal map, $f$, together with such a set $\left\{s_{i \lambda}\right\}$, determines a unique element, $\psi f \in h_{\pi}$, such that $\theta \psi f \in \rho_{2}$ is represented by $f$. Moreover, given $a \in h_{\pi}$, there is a normal map, $f_{a}: I^{2} \rightarrow X$, such that $a=\psi f_{a}$.

Let $a \in \theta^{-1}(0)$ and let $f_{a}: I^{2} \rightarrow X$ be a normal map such that $a=\psi f_{a}$. Let $A^{3}=p^{*} I^{2}$ be the join of $I^{2}$ with a new point $p^{*}$ and let $E^{2}=p^{*} \partial I^{2}$ $\subset \partial A^{3}$. Since $f_{a}$ represents $\theta a=0$ it may be extended to a map

$$
f:\left(A^{3}, E^{2}\right) \rightarrow\left(X, X_{0}\right) .
$$

We assume that $f^{-1} \sigma_{\lambda}^{2}$, for each $\lambda$, is a simplicial complex, which is mapped by $f$ simplicially on $\sigma_{\lambda}^{2}$. Then

$$
L=f^{-1}\left\{q_{\lambda}\right\}
$$

is a polygonal linkage, where $q_{\lambda} \in e_{\lambda}^{2}$. We assume that the vertices of $L$ are in general position, relative to the projection of $L$ from $p^{*}$ on $I^{2}$. Finally we assume that the segment $p^{*} I^{0}$ is mapped by $f$ on $x_{0}$. All these assumptions can be justified by geometrical arguments of standard type.

Let $G=\pi_{1}\left(A^{3}-L\right)$ with $p^{*}$ as base point. Then we define the homomorphism $F: G \rightarrow h_{\pi}$ as on pp. 423,424 of [2]. Let $g \in G$ be the element which is represented by $\partial I^{2}$, joined to $p^{*}$ by the segment $p^{*} I^{0}$. Assume that $a=F g$. Since $\partial I^{2}=\partial E^{2}$ and $E^{2} C A^{3}-L$ it follows that $g=1$, whence $a=0$. Therefore $\theta$ is an isomorphism. The proof that $F g=\psi f_{a}=a$ is the final stage in the proof of Theorem 4 in [2].

17. The groups $\pi_{n}\left(K^{n}\right)$. The methods and results in this paper can be carried further, in certain special cases at least, by introducing the system of groups, $\alpha_{n}(K)=\pi_{n}\left(K^{n}\right)$, associated with a given complex $K$. We then have the combined system $(\rho, \alpha)=\left\{\rho_{n}, \alpha_{n}\right\}$, where $\rho_{n}=\rho_{n}(K), \alpha_{n}=\alpha_{n}(K)\left(\rho_{1}=\alpha_{1}\right)$, with the homomorphisms

$$
\rho_{n+1} \stackrel{\beta}{\rightarrow} \alpha_{n} \stackrel{j}{\rightarrow} \rho_{n}
$$

We define a homomorphism, $(f, g):(\rho, \alpha) \rightarrow\left(\rho^{\prime}, \alpha^{\prime}\right)$, into another such system, as a family of operator homomorphisms, $f_{n}: \rho_{n} \rightarrow \rho_{n}^{\prime}, g_{n}: \alpha_{n}$ 
$\rightarrow \alpha_{n}^{\prime}$, associated with $f_{1}$, such that $j_{n} g_{n}=f_{n} j_{n}, g_{n} \beta_{n+1}=\beta_{n+1} f_{n+1}$. We define two such homomorphisms, $(f, g)$ and $\left(f^{*}, g^{*}\right)$, as equivalent, and write $(f, g) \simeq\left(f^{*}, g^{*}\right)$, if, and only if, there is a deformation operator, $\xi: \rho \rightarrow \rho^{\prime}$, associated with $f_{1}$, and an element $w^{\prime} \in \alpha_{1}^{\prime}$, such that

(a) $\quad w^{\prime} f_{n}^{*}-f_{n}=d_{n+1} \xi_{n+1}+\xi_{n} d_{n}$,

(b) $w^{\prime} \alpha_{n}^{*}-\alpha_{n}=\beta_{n+1} \xi_{n+1} j_{n}$.

It is easily verified that a (cellular) map, $K \rightarrow K^{\prime}$ in a complex $L$, induces a homomorphism $(\rho, \alpha) \rightarrow\left(\rho^{\prime}, \alpha^{\prime}\right)$, where $\rho^{\prime}=\rho\left(K^{\prime}\right), \alpha^{\prime}=\alpha\left(K^{\prime}\right)$. Let $\phi_{t}: K \rightarrow L$ be a (cellular) homotopy, such that $\phi_{0} e^{0}=\phi_{1} e^{0}=e^{\prime 0}$, where $e^{0} \in K^{0}, e^{\prime 0} \in K^{\prime 0}$ are the base points. Let $w^{\prime} \in \alpha_{1}^{\prime}$ be the element which is represented by the map $\theta: I \rightarrow K^{\prime 1}$, where $\theta(t)=\phi_{t} e^{0}$. Let $\xi: \rho \rightarrow \rho^{\prime}$ be the deformation operator, which is determined by $\phi_{t}$. That is to say, if $a \in \rho_{n}$ is represented by

$$
\lambda:\left(I^{n}, \partial I^{n}, I^{0}\right) \rightarrow\left(K^{n}, K^{n-1}, e^{0}\right),
$$

then $\xi a \in \rho_{n+1}^{\prime}$ is represented by $\mu: I^{n+1} \rightarrow K^{\prime}$, where

$$
\mu\left(t, t_{1}, \cdots, t_{n}\right)=\phi_{t} \lambda\left(t_{1}, \cdots, t_{n}\right) .
$$

Let $(f, g)$ and $\left(f^{*}, g^{*}\right)$ in (17.1) be the homomorphisms induced by $\phi_{0}, \phi_{1}$. Then (16.1a) may be verified directly, or deduced from the proof of Theorem 13 in $\$ 13$, and the concluding remarks in $\$ 10$. Let $\lambda\left(\partial I^{n}\right)=e^{0}$ in (17.2), and let $\lambda$ be interpreted as a map representing a given element $b \in \alpha_{n}$. Then $\mu$, given by (17.3), represents $\xi_{n+1} j_{n} b$ and (17.1b) follows without difficulty from (12.1) and the fact that $\mu\left(t \times \partial I^{n}\right)=\theta(t)$.

The value of this scheme is restricted by the fact that, in general, we know practically nothing about $\pi_{n}\left(K^{n}\right)$. But suppose that the groups $\alpha_{1}, \cdots, \alpha_{k}$ have been calculated, for some $k>2$. Then we can ignore the groups $\alpha_{n}$ for $n>k$ and, in some cases, the results in $\S \S 6,7$ above can be improved with the help of the system $\left(\rho, \alpha_{1}, \cdots, \alpha_{k}\right)$. For example, $j_{n}: \alpha_{n} \approx d_{n}^{-1}(0)$ for $n=1, \cdots, m$ if $K$ is a $J_{m}$-complex. In this case the groups $\alpha_{1}, \cdots, \alpha_{m}$ do not give us anything new and the interest begins with $\alpha_{m+1}$. Let $K$ be a finite complex and let $\pi_{n}(K)=0$ for $n=1, \cdots, m-1$. Further assume that $K^{m+2}$ is a reduced complex, as defined in [7]. Then we can calculate ${ }^{33}$ $\alpha_{m+1}$, and also $\alpha_{m+2}$ if $m \geqq 4$.

${ }^{33}$ See $\S 11$ in [6], Theorem 8 on p. 265 of [5], Lemma 4 on p. 418 of [2] and $\$ 12$ in [12]. 


\section{REFERENCES}

1. J. H. C. Whitehead, Combinatorial homotopy. I, Bull. Amer. Math. Soc. vol. 55 (1949) pp. 213-245.

2. —, Ann. of Math. vol. 42 (1941) pp. 409-428.

3. —_ Ann. of Math. vol. 42 (1941) pp. 1197-1239.

4. - Proc. London Math. Soc. vol. 45 (1939) pp. 243-327.

5. - Proc. London Math. Soc. vol. 48 (1944), pp. 243-291.

6. - Comment. Math. Helv. vol. 22 (1949) pp. 48-92.

7. - Annales de la Société Polonaise de Mathematique vol. 21.

8. A. L. Blakers, Ann. of Math. vol. 49 (1948) pp. 428-461.

9. S. Eilenberg and Saunders MacLane, Ann. of Math. vol. 43 (1942) pp. 757-831.

10. - Ann. of Math. vol. 48 (1947) pp. 326-341.

11. S. Eilenberg and N. E. Steenrod, Foundations of algebraic topology, not yet published.

12. R. H. Fox, Ann. of Math. vol. 49 (1948) pp. 471-510.

13. K. Reidemeister, Topologie der Polyder, Leipzig, 1938.

14. H. Seifert und W. Threlfall, Lehrbuch der Topologie, Leipzig, 1934.

15. W. Franz, J. Reine Angew. Math. vol. 185 (1943) pp. 65-77.

16. H. Hopf, Math. Ann. vol. 104 (1931) pp. 637-665.

17. H. Freudenthal, Compositio Math. vol. 5 (1937) pp. 299-314.

Magdalen College, Oxford University 